\title{
¿

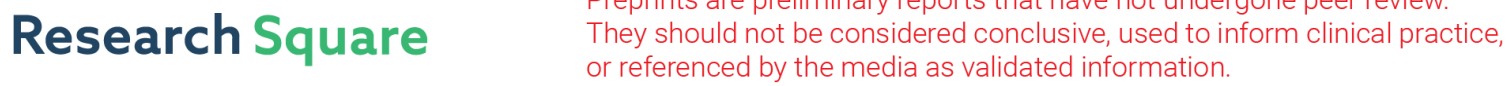 \\ Pi-starvation induced transcriptional changes in barley revealed by a comprehensive RNA-Seq and degradome analyses
}

\section{Pawel Sega}

Uniwersytet im Adama Mickiewicza w Poznaniu Wydzial Biologii https://orcid.org/0000-0001-59207514

\section{Katarzyna Kruszka}

Uniwersytet im Adama Mickiewicza w Poznaniu Wydzial Biologii

\section{Dawid Bielewicz}

Uniwersytet im Adama Mickiewicza w Poznaniu Wydzial Biologii

\section{Wojciech Karlowski}

Uniwersytet im Adama Mickiewicza w Poznaniu Wydzial Biologii

\section{Przemyslaw Nuc}

Uniwersytet im Adama Mickiewicza w Poznaniu Wydzial Biologii

\section{Zofia Szweykowska-Kulinska}

Uniwersytet im Adama Mickiewicza w Poznaniu Wydzial Biologii

Andrzej Pacak ( $\sim$ apacak@amu.edu.pl)

Uniwersytet im Adama Mickiewicza w Poznaniu Wydzial Biologii https://orcid.org/0000-0002-69689307

\section{Research article}

Keywords: Phosphate regulatory network, Barley, Small RNAs, Degradome, RNA-Seq

Posted Date: September 30th, 2020

DOI: https://doi.org/10.21203/rs.2.24665/v2

License: (c) (i) This work is licensed under a Creative Commons Attribution 4.0 International License. Read Full License

Version of Record: A version of this preprint was published on March 9th, 2021. See the published version at https://doi.org/10.1186/s12864-021-07481-w. 


\section{Abstract}

Background: Small RNAs (sRNAs) are 20-30 nt regulatory elements which are responsible for plant development regulation and participate in many plant stress responses. Insufficient inorganic phosphate (Pi) concentration triggers plant responses to balance the internal Pi level.

Results: In this study, we describe Pi-starvation-responsive small RNAs and transcriptome changes in barley (Hordeum vulgare L.) using Next-Generation Sequencing (NGS) RNA-Seq data derived from three different types of NGS libraries: (i) small RNAs, (ii) degraded RNAs, and (iii) functional mRNAs. We find that differentially and significantly expressed miRNAs (DEMs, $p$-value $<0.05$ ) are represented by 162 (44.88\% of total differentially expressed small RNAs) molecules in shoot and $138(7.14 \%)$ in root; mainly various miR399 and miR827 isomiRs. The remaining small RNAs (i.e., those without perfect match to reference sequences deposited in miRBase) are considered as differentially expressed other sRNAs (DESs, $p$-value Bonferroni correction < 0.05). In roots, a more abundant and diverse set of other sRNAs (DESs, 1796 unique sequences, $0.13 \%$ from total unique reads obtained under low-Pi) contributes more to the compensation of low-Pi stress than that in shoots (DESs, 199 unique sequences, $0.01 \%$ ). More than $80 \%$ of differentially expressed other sRNAs are up-regulated in both organs. Additionally, in barley shoots, up-regulation of small RNAs is accompanied by strong induction of two nucleases (S1/P1 endonuclease and 3'-5' exonuclease). This suggests that most small RNAs may be generated upon endonucleolytic cleavage to increase the internal Pi pool. Transcriptomic profiling of Pi-starved barley shoots identifies 98 differentially expressed genes (DEGs). A majority of the DEGs possess characteristic Pi-responsive cis-regulatory elements (P1BS and/or PHO element), located mostly in the proximal promoter regions. $\mathrm{GO}$ analysis shows that the discovered DEGs primarily alter plant defense, plant stress response, nutrient mobilization, or pathways involved in the gathering and recycling of phosphorus from organic pools.

Conclusions: Our results provide comprehensive data to demonstrate complex responses at the RNA level in barley to maintain Pi homeostasis and indicate that barley adapts to Pi-starvation through elicitation of RNA degradation. Novel P-responsive genes were selected as putative candidates to overcome low-Pi stress in barley plants.

\section{Background}

Barley (Hordeum vulgare L.) is one of the most commonly cultivated crop plants worldwide. It is a diploid plant with a low chromosome number $(n=7)$ and large genome size (haploid genome size of $~ 5.3 \mathrm{Gbp})$. In recent years, many resources essential to barley genomic studies have been developed, including a barley genome assembly in Ensembl Plants [1], a large number of expressed sequence tags (ESTs) [2], DNA markers, and useful techniques for stable or transient transformation of barley [3]. The simplicity of cross-breeding and cultivation in a wide range of climatic conditions makes barley a model crop plant in the study of desirable agronomic traits [4]. Studies on the responses of barley to abiotic stresses can help to better its cultivation in variable and adverse conditions. Environmental stressors cause crop damage 
and reduction of yields, which result in financial losses for agricultural businesses. In plants, abiotic stresses trigger specific stress-induced molecular pathways that often involve different classes of small RNAs (sRNAs) [5-7].

Small RNAs (sRNA) are non-translating into protein class of RNA (20 -30 nt) [8]. Best known are siRNA (small interfering RNAs) and miRNA (microRNAs, 18-25 nt) - a class of double-stranded RNA which may target chromatin or transcripts to regulate both the genome and transcriptome [9, 10]. Plant small RNAs tend to bind to Argonaute (AGO) family proteins to form either RNA-induced silencing complexes (RISC) for post-transcriptional gene silencing (PTGS) [11] or RNA-induced initiation of transcriptional silencing (RITS) complex for transcriptional gene silencing [12]. Recently, many studies have emerged about various sRNA types, biogenesis, targets, and functions [13-15]. Based on the biogenesis pathway, small RNAs have been classified into miRNAs, siRNAs, phasiRNA and tRFs (tRNA-derived RNA fragments) [16]. Among them, miRNAs and siRNAs are the most extensively studied sRNAs in plants.

Plant MIR genes represent independent transcriptional units, which are transcribed by RNA polymerase II (RNA Pol II). Primary transcripts (pri-miRNAs) maturate in a two-step process in the cell nucleus [17]: Firstly, pri-microRNAs are diced out by the DCL1 (믙ER-LIKE 1) protein from a stem-loop precursors [18]. The next step of DCL1 protein action leads to the generation of a double-stranded molecule composed of a guide miRNA strand and the passenger miRNA* (star) strand (called the miRNA/miRNA* duplex). Different DCL family members produce miRNA molecules of different lengths; however, the majority of plant miRNAs are 21 nucleotides in length [19]. The miRNA is assembled together with AGO1 (ARGONAUTE 1), in order to create RISC in the cytoplasm which is responsible for mRNA slicing. The cleavage position is precisely determined and occurs in the target mRNA between nucleotides complementary to the $10^{\text {th }}$ and $11^{\text {th }}$ nucleotides of the related miRNA, counting from the miRNA's $5^{\text {'-end }}$ [20]. Ultimately, target mRNA recognized by the specific miRNA molecule is degraded by 5'-to-3' exonucleases and the overall pool of valid mRNA transcripts is decreased [21]. Such a mechanism exists in plants to modulate the expression levels of crucial stress-responsive genes [22].

In plants, there are many types of siRNAs, including (i) nat-siRNAs (natural-antisense siRNAs), which are produced from overlapping regions of natural sense-antisense mRNA pairs; (ii) ta-siRNAs (trans-acting siRNAs), processed from non-coding RNA precursors; and (iii) ra-siRNAs (repeat-associated siRNAs), generated from transposable and repetitive elements to mediate further steps of RNAi $[9,23]$. tRFs may be produced after cleavage of tRNA ends (to generate 5'-tRF and 3'-tRF) by RNAse T2 [24], as well as DCL (DICER-LIKE) processing in plants [25]. Both miRNAs and siRNAs mediate RNA interference (RNAi) in plants, but there are subtle differences between them. As an endogenous molecule miRNA is diced-out from microRNA precursor folded in stem-loop structure [26], while siRNA is a double-stranded RNA derived from the host genome or directly from viruses or transgenes [27].

The expression of sRNAs changes in response to environmental factors [7, 28] or viral infection [29-31]. Mentioned above classes of sRNAs appear to play important roles in plant growth, development regulation, and adaptation to various stresses. In barley, miRNAs have been shown to (i) mediate 
tolerance to heat stress [32], (ii) confer drought tolerance [33], (iii) regulate low-potassium tolerance [34], (iv) respond to aluminum stress [35], and (v) maintain inorganic phosphate (Pi) homeostasis [36]. On the other hand, siRNAs mostly function as a defenders of genome integrity in response to foreign nucleic acids [37]. The TAS3 gene expresses ta-siRNAs, which may negatively regulate auxin signaling by targeting $\underline{A} U$ UXIN $\underline{R} E S P O N S E$ FACTOR $\underline{3}$ (ARF3) transcripts [38] and moderate floral architecture in response to drought stress in Arabidopsis thaliana L. [39]. The TAS-ARF pathway has been shown to be involved either in the development process of maize (Zea mays L.) [40] or regulating lateral root growth in Arabidopsis [41]. In addition, tRNA-derived small RNAs have been shown to accumulate in Arabidopsis roots under Pi-starvation [42], while rhizobial tRFs can regulate nodule formation in soybean (Glycine max L.) [13].

Changes in soil nutrient concentrations lead to aberrations in the set of sRNAs, with respect to the prevailing severe environmental conditions [6]. One of the most important macronutrients, which is indispensable for proper plant growth, is phosphorus $(P)[43,44]$. $P$ is a component of DNA, RNA, phospholipids, and ATP, and is involved in several biochemical processes such as protein phosphorylation, energy storage and transfer, and regulation of protein synthesis [45]. From soil matrices, $\mathrm{P}$ is acquired by the root system in the form of inorganic phosphate (Pi) ions. Insufficient Pi supply leads to barley growth inhibition [46, 47]. Plant transcriptome response to Pi-starvation involves protein coding genes, sRNAs, and long non-coding RNAs that form regulatory feedback loops. The most widely studied molecules in this context-miRNA399 molecules-are up-regulated in barley shoots and roots under lowPi conditions [36]. MiRNA399 targets the 5'-UTR of the barley PHO2 ( encoding an ubiquitin-conjugating E2 enzyme (UBC24), a negative regulator of Pi uptake and root-toshoot translocation. PHO2 is involved in ubiquitination of PHOSPHATE IRANSPORTER 1 (PHT1) family [49] and PHOSPHATE TRANSPORTER TRAFFIC EACILITATOR 1 (PHF1) [49]. Transgenic Arabidopsis thaliana plants overexpressing miR399 accumulate excessive $\mathrm{Pi}$ in shoots and display Pi overaccumulation toxic symptoms. Likewise, such a phenotype has been reported for the pho2 loss-offunction Arabidopsis mutant [50,51]. Thus, plants have developed a strategy to regulate the level of miR399 in the cytoplasm. The non-coding RNA molecule, IPS1 (INDUCED BY PHOSPHATE STARVATION 1), has been shown to be highly expressed in plants exposed to Pi-starvation [52-54]. IPS1 is a noncleavable miR399 target which inhibits miR399-mediated down-regulation of PHO2 mRNA by target mimicry [54]. Thus, the RNAi effect of miRNA activity may be counterbalanced by other RNAs, in a stressdependent manner.

Deep sequencing of sRNAs has uncovered up-regulation of miRNAs like miR156, miR778, miR827, and miR2111, and down-regulation of miR169, miR395, and miR398 in Arabidopsis plants upon Pi deprivation [42, 55]. In rice (Oryza sativa L.), Pi-starvation induced the expression level of miR827 molecules, which dysregulate the transcript level of two genes encoding the SPX-MFS (named after

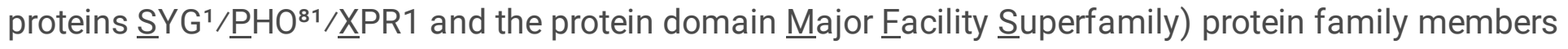
SPX-MFS1 and SPX-MFS2 [56, 57]. These two SPX-MFS membrane transporters mediate Pi transport and control Pi homeostasis in shoot [58]. In Arabidopsis, the level of mature miR778 was up-regulated in shoots and roots in low-Pi conditions, while its target gene expression SUVH6 (느(VAR)3-9 $\underline{H O M O L O G} \underline{6})$ 
was accordingly reduced [59]. The SUVH6 gene encodes a histone H3 lysine 9 (H3K9) methyltransferase, which may enable plants to adapt to environmental conditions by changing their chromatin structure [60]. miR2111 functions as an activator of rhizobial nodulation, which is strictly correlated with the balanced assimilation of nitrogen $(\mathrm{N})$ and $\mathrm{P}$ in plants [61, 62]. However, there is still a gap in understanding how Pistarvation affects the quantity and quality of sRNAs distributed in barley shoots and roots. What kind of sRNAs are preferentially induced? What is the role of sRNAs in responding to Pi-starvation? What are the mRNA targets recognized by those sRNAs in barley?

In this paper, we analyzed changes in the expression levels of RNAs in barley growing under Pi-starvation, as compared to control/Pi sufficient conditions. Our results support the hypothesis that Pi-starvation triggers underlying molecular mechanisms and the expression level of key genes involved in maintaining proper barley growth and development. Combined deep sequencing data (sRNAs, degradome and mRNAs) reveals the widespread importance of low-Pi-dependent miRNAs and genes representing various biological pathways. Using degradome analysis, we identified mRNAs targeted by sRNAs identified in this study. Among these sRNAs, only a small fraction maps perfectly to miRNA sequences deposited in miRBase. Our degradome data show that most sRNAs produced upon Pi-starvation are not involved in gene silencing. In addition, we performed transcriptome analysis of the protein-coding gene expression in barley shoots upon Pi-starvation. Subsequent analyses were performed (GO analysis, chromosomal mapping, and Pi-responsive motifs localization) to characterize specific stress responses in barley plants to accomplish Pi homeostasis.

\section{Results}

\section{Barley plants display low-Pi symptoms at the morphological and molecular levels}

Severe low-Pi responses were induced in the barley plant line Rolap grown in the soil containing $8 \mathrm{mg}$ $\mathrm{P} / \mathrm{kg}$. P undernourishment caused over 2-fold reduction of plant shoot biomass (Fig. 1A). Shoot fresh weight of plants at $23^{\text {rd }}$ day post-sowing (dps) was significantly reduced, in comparison with control plants, with average mass $8.8 \mathrm{~g}$ for stressed plants and $18.5 \mathrm{~g}$ for plants growing under Pi-sufficient conditions $(p=0.001)$ (Fig. 1B). We observed a significantly decreased concentration of $\mathrm{Pi}$ ions, with only $0.48 \mu \mathrm{mol}$ Pi per g of fresh root weight (FW) and $4.2 \mu \mathrm{mol}$ Pi per g of shoot FW, when compared with the control plants having $3.84(p=0.0056)$ and $24.35 \mu \mathrm{mol} \mathrm{Pi} / \mathrm{g} \mathrm{FW}(p=0.0001)$, respectively (Fig. 1C). To examine the induction of changes at a molecular level by low-Pi stress in barley plants, we measured the absolute gene expression of the low-Pi-responsive marker gene IPS1. The barley IPS1 gene is highly expressed under Pi-deficient conditions in the plant line Rolap. At the tillering stage (23 dps), we detected 4191 copies of IPS1 RNA for low-Pi treated roots, normalized per 1000 copies of $\underline{A}$ DP-RIBOSYLATION FACTOR 1-LIKE (ARF1) reference gene, in comparison to the control plants, with only 58 copies of IPS1 RNA $(p=0.00006)$ (Additional file 1). Taking validated plant material, we performed tripartite deepsequencing analysis to: (i) identify Pi-responsive sRNAs, (ii) elucidate changes in the barley transcriptome upon Pi starvation, and (iii) identify mRNA targets for Pi-responsive sRNAs through degradome sequencing (Fig. 2). 


\section{Barley plants express an organ-specific set of microRNAs in response to low-Pi conditions}

We performed small RNA deep-sequencing to find out which small RNAs are up- or down-regulated by Pi starvation in barley shoots and roots. The average of $30.4 \mathrm{mln}$ reads for roots and $25.2 \mathrm{mln}$ reads for shoots were generated in $50 \mathrm{nt}$ single-read Illumina sequencing (Additional file 2). After adapter and quality trimming, we mapped reads to the miRBase Sequence Database (release 22) to annotate miRNAderived sequences [63]. A set of parameters were used to define the pool of differentially expressed miRNAs: (i) no mismatches with the reference sequences in the miRBase were allowed; (ii) different types of miRNA sequences were permitted, whether they were annotated as precursor, mature, or isomiR; (iii) miRNA sequences were named accordingly to the name of the assigned reference miRNA; and (iv) significance of fold change ( $p$-value $<0.05)$ was additionally verified using a restricted Bonferroni $p$-value test (Fig. 2).

We found 138 and 162 differentially expressed miRNAs (DEMs) annotated to the miRBase ( $p$-value < 0.05 ) in barley roots and shoots, respectively. Only 25 DEMs were expressed in both examined barley organs (Additional file 3). However, restricted Bonferroni $p$-value correction narrowed down set of miRNAs to 15 in shoots and 13 in roots (Fig. 3A, left panel). We focused further only on those that passed the Bonferroni test (Fig. 3A, right panel). In both organs, most of the DEMs were significantly up-regulated. Interestingly, out of 15 miRNA, only miRNA166d was down-regulated in shoot under low-Pi $\left(\log _{2}\right.$ (fold change) $=-1.18$ ). In our previous work, we showed that miRNA166 is expressed in barley during different developmental stages reaching the highest level in 2-week-old plants [64]. miRNA166 plays an important role in plant development, including root and leaf patterning, by targeting mRNA encoding HOMEODOMAIN LEUCINE-ZIPPER CLASS ㅆI (HD-ZIP III) transcription factors [65]. Similarly, only miRNA319b out of 13 DEMs was down-regulated in low-Pi treated roots $\left(\log _{2}\right.$ (fold change) $\left.=-1.28\right)$. In a previous study, we presented data that Arabidopsis miR319 is a multi-stress responsiveness miRNA [22]. For example, MIR319b gene expression was down-regulated in response to drought, heat, and salinity, but up-regulated in response to copper and sulfur deficiency stresses [22].

\section{Uncharacterized miRNAs are highly induced by low-Pi stress in barley roots}

A specific set of miRNAs was expressed in barley shoot or root under low-Pi (Additional file 3). In shoot, only two miRNA families, miRNA399 and miRNA827, were induced, while in root we observed a more diverse response (Fig. 3A, right panel). Apart from miRNA399/miRNA827 induction, we found the following additional miRNA to be up-regulated in root: two miRNA5083 (id $=3$, and id $=4$ ), miRNA1511 (id = 6), two miRNA9779 (id = 16, and id = 17), two miRNA156 (id = 65, and id =69), and miRNA5072 (id = 118). Among these eight miRNAs, only miR 156 has been reported before as Pi-responsive in Arabidopsis $[42,55]$. The miR1 56 isomiRs were also found dysregulated in shoot, but none of them pass the Bonferroni test. Our results suggest that there is a more complex response to low-Pi stress regarding miRNA expression in roots than in shoots, where the miRNA action is directed to control the transcript level of either $P H O 2$, SPX-MFS1, or SPX-MFS2 by just two miRNA families. 
In order to determine the potential cleavage activity of miRNAs identified in root we performed degradome analysis. None of these eight miRNAs were found to recognize a specific target from the pool of barley mRNAs. Our result raises the hypothesis that plants may release Pi from the pool of stress-responsive small RNAs. In addition, most of them match to the pre-miRNAs, not mature molecules (Fig. 3A, right panel).

NGS (Next Generation Sequencing) data were validated by complex analysis of mature miR827 (from 3' arm) in all samples taken for deep sequencing. The absolute expression level of miR827 is significantly up-regulated in both shoots and roots under a low-Pi regime (Fig. 3B). The $\log _{2}$ fold change of miR827 molecules defined by deep-sequencing in shoot (id: 2073) was found on the same level in root (id: 114), $\log _{2}(f c)=3.05$ and 3.01, respectively (Fig. 3A). The ddPCR results were consistent with NGS data showing up-regulation of mature miR827 molecule in both tested organs (Fig. 3B). These data were confirmed by northern blot hybridization (Fig. 3C) and degradome analysis (Fig. 3D). The results showed that SPX-MFS1 transcripts have been recognized and cleaved by Ago protein associated with miR827 in barley $(p=0.014)$ (Fig. 3D).

\section{Different classes of small RNAs in barley accumulate in an organ-specific manner under low-Pi regime}

The small RNA reads which did not map to miRBase were mapped to particular classes of barley cDNAs derived from the Ensembl Plants database (release 40), both separately and to all of them (Fig. 2). All sequences mapped to barley cDNAs are listed in Additional file 3. We found that small RNAs, other than miRs, differentially expressed (DESs) in barley under Pi starvation were represented by 199 unique sequences ( $0.01 \%$ of all unique reads from barley shoots (Additional file 4$)$ and by $1796(0.13 \%)$ unique reads from roots (Fig. 4A, Additional file 5).

We analyzed whether different lengths (taking sequences from 18 to $25 \mathrm{nt}$ in lenght) and classes of small RNAs contributed to either root or shoot response to low-Pi conditions. In roots, the length distribution of DESs remained balanced, from $10.91 \%$ for the representation of $24 \mathrm{nt}$ sequences to $15.26 \%$ for the $18 \mathrm{nt}$ sequences, which were the most abundant (including 274 DESs) (Fig. 4B). In shoots, the representations of DES lengths fluctuated more than in roots. The $19 \mathrm{nt}$ sequences were the most visible $(21.11 \%)$, while three representations did not score more than 10\%: the $22 \mathrm{nt}(9.55 \%), 23 \mathrm{nt}(8.54 \%)$, and $25 \mathrm{nt}(3.52 \%)$ sequences (Fig. 4B, Additional file 6).

In roots, 1070 unique reads were mapped to cDNA sequences annotated in the Ensembl Plants databases (non-translating, protein-coding, pseudogenes, rRNA, snoRNA, snRNA, sRP-RNA, tRNA), while 726 unique sequences remained without match. The DESs obtained from low-Pi roots were mostly annotated to protein-coding mRNAs (38.54\%), rRNAs (34.17\%), and non-translating RNAs (19.49\%). Below $5 \%$ of overall DESs, we found a number of remaining cDNA classes, such as snoRNAs (2.49\%), tRNAs (2.47 \%), SRP-RNAs (1.17\%), snRNAs (0.95\%), and pseudogenes (0.65\%). While in shoot, we found 199 DESs under the low-Pi regime. Altogether, 116 out of 199 differentially expressed small RNAs (DESs) were annotated to the barley Ensembl Plants database, where 83 sequences remained without match 
(Additional file 4). In the case of shoot samples, $85 \%$ of annotated DESs represented only protein-coding mRNAs (47.87 \%) and non-translating RNAs (36.49\%) (Fig. 4A, right panel; Additional file 7). We did not find any DESs annotated to the snRNAs, SRP-RNAs, or tRNAs from barley shoot upon low-Pi. In addition, total numbers of 166 DESs (83 \%) in shoots and 1560 DESs (87\%) in roots were significantly upregulated after exposure to low-Pi stress (Additional file 4, Additional file 5, Fig. 7).

Among the unannotated reads of sRNAs in roots, the highest fold change was observed for a 19 nt 5'ACCTACTCGACCTCGGCCG-3' molecule ( $\log _{2}$ (fold change) $=8.02$, induction) and a 22 nt 5 '-

CTAATACCGGATACGCGAACCG-3' molecule (-5.87, repression). The BLAST (Basic L Local Alignment Search Iool) analysis of first (19 nt) molecule showed a perfect match to either the intergenic region of barley chromosome no. 5 , soil bacteria (mesorhizobium), or Linum usitatissimum L., while the second molecule (22 nt) mapped to RNA encodes 16S rRNA. Furthermore, in roots, the most abundant small RNA was a 25 nt 5'-ACCGACCTACTCGACCCTTCGGCCG-3' molecule (15847.7 and 65590.5 mean of normalized counts in barley root in control and low-Pi conditions, $\left.\log _{2}(f \mathrm{c})=2.82\right)$. This small RNA matched several barley loci encoding SSU (small subunit) rRNAs (Additional file 5).

In our results from low-Pi treated shoot samples, the highest fold change was represented by a 24 nt 5'AAGATTGGTTGGTTGGTTGGGTCT-3' molecule ( $\log _{2}(\mathrm{fc})=8.72$, induction). This $24 \mathrm{nt}$ molecule is a part of transcript encoding a putative pentatricopeptide repeat (PPR) protein. The PPR protein family facilitates the processing, splicing, editing, stability, and translation of RNAs in plants [66]. The most abundant small RNA was a 19 nt 5'-GGGCCTGTAGCTCAGAGGA-3' molecule (9471.5 and 49914.1 normalized mean counts in barley shoot in control and low-Pi, respectively, $\left.\log _{2}(\mathrm{fc})=2,45\right)$. This sRNA was mapped to the barley genomic loci (EPIHVUG00000039813), which encodes arginyl-tRNA (trnR-ACG) and a cDNA encoding uncharacterized protein (HORVU2Hr1G084630) which is likely involved in carbon fixation. Interestingly, the pool of DESs was selective, considering organ-specific expression change, providing only three unique sequences that were significantly changed in both barley organs under low-Pi regime (Fig. 4A, left panel). These molecules were: (i) 20 nt 5'-AGTAGAGGTCGCGAGAGAGC-3' $\left(\log _{2}(\mathrm{fc})=\right.$ 2.01 in root and 1.16 in shoot, respectively) annotated to the $26 \mathrm{~S}$ rRNAs, (ii) 24 nt 5'-

ATTCTCCGCGTCGGATACCTGAGA-3' (3.69 in root and 2.07 in shoot) encoding the barley MYB21 transcription factor, and (iii) 21 nt 5'-TGCCAAAGGAGAACTGCCCTG-3' (4.64 in root and 6.27 in shoot) mapped to the intergenic region of barley chromosome no. 3 (Additional file 4).

The results obtained in this study show again that barley roots exhibit a more diverse pool of Piresponsive small RNAs which may trigger developmental adaptation of the root to Pi-starvation. Additionally, 613 rRNA-derived sRNAs are up-regulated, whereas 176 rRNA-derived sRNAs are downregulated in barley roots (Additional file 5). We believe that such sRNA may be further processed, serving as a Pi source to compensate Pi deficiency.

\section{Identification of barley genes responsive to Pi-starvation}


Since we observed, that most of the other sRNAs in shoot were derived from either protein-coding mRNAs or non-translating RNAs, we checked whether this observation is correlated with gene expression changes of polyadenylated RNAs in barley shoot under Pi-starvation. Among 98 of identified DEGs, the transcripts of 56 annotated loci were significantly up-regulated, while those derived from 42 loci were down-regulated in Pi-starved barley shoots (Additional file 8). Repressed loci were found to be preferentially located at barley chromosome no. 2, while induced loci were found mostly at barley chromosomes no. 3 and 5 (Fig. 5B).

The highest enrichment of DEGs was found in the GO terms, either (i) belonging to the cellular components of the chloroplasts; (ii) showing catalytic activity, either ion or metal binding properties; and (iii) involved in the various biological and metabolic processes related to stress response and plant defense (Fig. 5A). A major set of up-regulated DEGs represented genes involved in the Pi signaling. Among them, we found genes encoding: IPS1 $\left(\log _{2}(\mathrm{fc})=5.89\right)$ [54], inorganic pyrophosphatase (PPase, 4.01) [67], SPX-domain containing protein $\underline{5}$ (SPX5, 3.44) [68], phosphate transporter PHOSPHATE 1-3 (PH01-3, 2.97) [69], SPX-MFS2 (2.79) [56], haloacid dehalogenase-like hydrolase (HAD1, 1.95), [70] and five different purple a acid phosphatases (PAPs) (Fig. 5C; Additional file 8) [71]. Interestingly, four genes were induced to a higher extent than the low-Pi stress marker, IPS1 gene. These genes encode ferredoxin $\left(F D 1, \log _{2}(f c)=14.20\right)$, mitochondrial-processing peptidase $(13.35)$, chlorophyll a/b binding protein (8.90), and alpha-amylase (7.30), and are engaged in photosynthesis, redox reactions, reactive oxygen species (ROS) homeostasis, and co-ordinated mobilization of nutrients. Chloroplasts and mitochondria are the organelles with the highest Pi requirements. Strong FD1 gene up-regulation most likely reflects the accumulation of reduced ferredoxin in chloroplasts. Low-Pi lowers the capacity to process incoming light and enhances starch accumulation in chloroplasts, thereby leading to photoinhibition $[72,73]$. Within the category of genes that were significantly down-regulated, most of them were related to stress and defense responses (Additional file 8); for instance, uncharacterized protein (HORVU2Hr1G030090, -6.50), oxalate oxidase (-4.41) [74], beta-sesquiphellandrene synthase (-3.41), glutamate carboxypeptidase (-3.17), chalcone synthase (-3.05) [75], or caleosin-like protein (-2.95) (Fig. 5C). Only two repressed genes are known to be directly involved in Pi signaling and metabolism, SPX-MFS1 (-2.58), targeted by miR827 [57] and probable inactive purple acid phosphatase (-1.75). Additionally, two genes encoding laccases ( LAC19-like, Additional file 8), cell wall-localized multi-copper oxidases, were significantly down-regulated (-2.10 and -2.44) in our mRNA RNA-Seq data. Laccases are involved in copper homeostasis (Fig. 7) and lignin biosynthesis, and have been shown to be targeted by miR397 in maize [76] and Arabidopsis [77]. Furthermore, key genes encoding proteins involved in the nitrate and phosphate cross-talk were affected by low-Pi conditions in barley shoots, such as NIGT1 (NITRATE-INDUCIBLE, GARP-TYPE IRANSCRIPTIONAL REPRESSOR 1) transcription factor $(3.80)[78,79]$ and nitrite reductase (1.98), as well as high-affinity nitrate transporter NRT2.1 (NITRATE IRANSPORTER 2.1) (-2.60) [80].

Absolute quantification of a few selected transcripts was performed to validate RNA-Seq data obtained in this study. Two genes which were highly induced (encoding endonuclease S1/P1 and 3'-5'-exonuclease) and two which were severely repressed (encoding oxalate oxidases) under the low-Pi regime were taken 
for ddPCR (droplet digital PCR) analysis (Fig. 6A). We confirmed statistically significant changes ( $p<$ 0.05 ) in normalized copy number (per 1000 copies of the ARF1 reference gene) of all genes taken for analysis.

\section{Pi-responsive motifs found in the promoters of DEGs}

In general, genes that are affected by Pi status possess characteristic cis-regulatory elements within either promoter or 5'-UTR regions [81]. Previously, we have shown the importance of the P1BS motif (PHR $\underline{1}$ binding sequence, consensus GnATATnC, [82]) and P-responsive PHO elements (consensus ATGCCAT, [83]) in the expression efficiency of the barley PHO2 gene [48]. Both motifs may bind PHR-like (PHOSPHATE STARVATION RESPONSE) transcription factors (TFs) and act as activators or repressors of downstream gene expression in a Pi-dependent manner [84]. Likewise, we hypothesized that regulatory regions of the identified DEGs had Pi-responsive motifs, which may be bound by PHR TFs, causing gene expression dysregulation. To confirm this hypothesis, we analyzed DNA sequences from the $2000 \mathrm{bp}$ region upstream of the predicted transcription start sites from all 98 DEGs (Additional file 9). In the next step, promoter data were directly screened for P1BS and P-responsive PHO element consensus sequences by multiple promoter analysis using the PlantPAN3.0 tool [85]. We confirmed the presence of Pi-dependent motif in 55 out of 98 DEGs promoters. An in silico approach detected 46 DEGs having at least one P1BS motif (Additional file 10) and 17 DEGs with at least one P-responsive PHO element (Fig. 6B, Additional file 11). The most over-represented motifs were found in the promoters of genes encoding sulfoquinovosyl transferase SQD2-like $\left(\log _{2}(\mathrm{fc})=3.74\right)$ [86], phosphoenolpyruvate carboxylase 1-like $\left(\log _{2}(f c)=1.73\right)$ [87], and pyridoxal phosphate-dependent transferase $\left(\log _{2}(f c)=-2.61\right)$ [88]. Each of the genes harbor three P1BSs and one P-responsive PHO element, as well (Additional file 8).

\section{Degradome profiling describes post-transcriptional regulatory network of identified DEMs}

After identification of (i) differentially expressed miRNAs (DEMs), (ii) other sRNAs (DESs), and (iii) mRNAs (DEGs), we used this comprehensive data together with cDNAs annotated in the Ensembl Plants database to identify the sRNAs directly involved in RNA degradation. The DESs were also examined, because we assumed that there may have been putative miRNAs that were not mapped to the miRbase, due to restricted query settings allowing no mismatch. Molecules which exhibited a single mismatch (or more) may still function as miRNA in barley. Degradome libraries were carried out for root, as well as for shoot, and sequenced using an Illumina System. The received data were analyzed using two independent in silico approaches (Fig. 2). At times, the different algorithms used elicited different miRNA targets; however, the general degradome pattern was equivalent for both approaches (Fig. 3A, right panel,

\section{Additional files 12-24).}

First, we searched for the potential target mRNAs for a broader set of differentially expressed miRNAs with significant fold change ( $p$-value without Bonferroni correction), taking 162 DEMs from shoot and 138 DEMs from root, respectively (Additional file 3). A total of 1964 scores were obtained for shoot DEMs (1303 using the TargetSeek approach and 661 using PAREsnip2) (Additional file 12, Additional file 16), 
while in root there were 1515 records (921 and 594, respectively) (Additional file 14, Additional file 20, Additional file 21). Accordingly, shoot examination proved the proper selection of miRNAs from all differentially expressed small RNAs, as all 661 records predicted by the PAREsnip2 approach were categorized with the best score (0). A majority of records corresponded to different miR399 and/or miR827 isomiRs and their known targets PHO2 or SPX-MFS1/SPX-MFS2, respectively. In shoot, the best scoring miRNA:mRNA match was found for mature miRNA399d (21 nt, id = 2066), which guides cleavage within the 5'-UTR of PHO2 mRNA (isoform no. 3) in position 857 (TargetSeek: score = 0, MFE = -41,1, PAREsnip2: score= 0, MFE = -41.1) (Table 1). The miR5049e (21 nt) targets various isoforms of mRNA encoding ENT domain-containing proteins with high probability (TargetSeek: score $=1.5, \mathrm{MFE}=-27.4$ ), which may be involved in the plant defense response to fungal infection and anthocyanin biosynthesis $[89,90]$. Moreover, we found two target mRNAs (HORVU3Hr1G094730, HORVU6Hr1G031450) encoding SPL-like TFs guided for cleavage by two miR156 isomiRs (id: 2053 and 2054) in barley shoot (Additional

file 16, Additional file 17). The $S P L$ genes are post-transcriptionally regulated by miR156 and control shoot branching in rice [91].

In contrast, in roots, where the pool of identified DEMs is more diverse, the PTGS regulatory network involves many TFs with various functional domains (Additional file 14). For instance, the $21 \mathrm{nt}$ mature miR444b (id = 101) targets mRNA encoding MADS-box transcription factor 57 (HORVU6Hr1G073040, TargetSeek: score $=0$, MFE $=-40.1$, PAREsnip2: score $=0$, MFE $=-40.1$, which regulates long-distance nitrate transport and root elongation in rice [92] (Table 1). The well-known abiotic stress-responsive miR319c (21 nt) [6] was found to target various isoforms of GAMyb TF (HORVU3Hr1G079490, TargetSeek: score $=5$, MFE $=-34.6$, PAREsnip2: score = 3, MFE = -35.4). The same predicted miR319 target has been previously found in RNA-Seq of Pi-starved tobacco plants [93]. Its targets, the plant GAMyb TFs, have been shown to activate gibberellin-responsive gene expression of a-amylase in barley $[94,95]$. Our degradome data indicate the possible cleavage of various mRNA isoforms encoding nuclear transcription factor $\underline{Y}$ subunit $\underline{A}-\underline{4}$ (NFYA4, HORVU4Hr1G005670, TargetSeek: score $=3$, MFE $=-27.6$, PAREsnip2: score $=2$, MFE $=-28.1)$ mediated by miR169d $(20 \mathrm{nt}$, id $=84)$ (Table 1), what is consistent with previous studies on Cunninghamia lanceolata L. showing that NFYA4 mRNA is targeted by miR169d (authors used PAREsnip approach) [96]. The NFYA4 protein is involved in many abiotic stress responses and may regulate the timing of transition from vegetative to reproductive phase [97]. All predicted DEM targets for both organs are listed in Additional files 13, 15, 17, and 21.

\section{Putative regulatory small RNAs identified in degradome data}

Degradome profiling was performed to test whether any of the sequences from the 1796 DESs found in roots or 199 DESs found in shoots contribute to the complexity of gene regulation during low-Pi stress. A total of 759 records (245 using the TargetSeek approach and 514 using PAREsnip2) were found in the degradome profiles matching root DESs (Additional file 15, Additional file 22, Additional file 23) and 160 records (87 and 73, respectively) matching shoot DESs (Additional file 13, Additional file 18, Additional file 19). Taking only either the most up-regulated or the most down-regulated sRNAs for degradome screening, we found six promising target genes in shoot and five in root (Table 2). For example, in roots, 
the highly up-regulated $20 \mathrm{nt}$ sequence 5'-ACCGACCTACTTGACCCTTC-3' $\left(\log _{2}(\mathrm{fc})=6.46\right.$, id $\left.=348\right)$ binds to the 3'-UTR region of the MYB44 TF's mRNA and guides/promotes cleavage in the 1037 position (PAREsnip2: score = 4; MFE = -33.3) (Table 2). RNA-Seq data for potato (Solanum tuberosum L.) proved that expression of the MYB44 gene is highly downregulated under low-Pi in roots [98], which may be the result of miRNA-guided PTGS. Studies in potato have indicated that MYB44 TF may form a regulatory complex together with WRKY6 TF, which negatively regulates Pi transport by suppressing PHO1 expression [98]. Other degradome records, among the most differentially expressed sRNAs, were found to target mRNAs of the V-ATPase assembly factor (VMA21-like) and three barley genomic loci encoding uncharacterized proteins (HORVU7Hr1G053570, HORVU1Hr1G027340, and HORVUOHr1G023910) (Table 2). For example, the potential cleavage activity was predicted for 24 nt sequence 5 'AGAGGAAACTCTGGTGGAGGCTCG-3' $\left(\log _{2}(\mathrm{fc})=-3.58\right.$, id = 463), which may target the mRNA encoding uncharacterized protein with unknown PTHR47188 domain (Fig. 4C).

Analogous degradome screening was done for shoot data. Among all identified DESs, we found that the most upregulated sequence, 24 nt 5'-AAGATTGGTTGGTTGGTTGGGTCT-3' $\left(\right.$ long $_{2}(\mathrm{fc})=8.72$, id = 2112), targets the $3^{\prime}$-UTR of mRNA encoding multiple organellar RNA editing factor $\underline{9}$ (MORF9, HORVU7Hr1G073170, TargetSeek: score $=16.5$, MFE $=-28.1)($ Table 2). MORF9 proteins are required for RNA editing in plastid mRNAs, which may contribute to stress adaptation in plants $[99,100]$. In both approaches, we found that the 21 nt sequence 5'-TGCCAAAGGAGAACTGCCCTG-3' $(6.27$, id $=2265)$ targeted the same isoform of PHO2 mRNA (HORVU1Hr1G085570.3, TargetSeek: score = 6, MFE $=-29.9$, PAREsnip2: score $=3.5$, MFE $=-33.4)$. When we browsed the miRBase using this 21 nt sRNA as a query, we found high similarity to the osa-miR399a, exhibiting only one mismatch. Thus, we suspect that such sRNA may function as another miR399 isomiR in barley. Most dysregulated DESs were also found to target mRNAs encoding methyltransferase type 11 domain-containing protein (MT11), AAA-ATPase (At3g50940-like), lysine-specific demethylase 5A (LSD), or an uncharacterized protein with a predicted transmembrane domain (HORVU3Hr1G036970). The best scoring records were found for the $21 \mathrm{nt}$ sequence 5'- TTAGATGACCATCAGCAAACT-3' (3.11, id = 2279), which targets mRNAs encoding SPX-MFS1 (PAREsnip2: score $=2.5$, MFE $=-27.5$ ) and SPX-MFS2 (TargetSeek: score $=3.5$, MFE $=-26)$ (Table 3). This result suggests that such sRNA may exist as another miR827 isomiR. Moreover, this is consistent with the screening made for differentially expressed miRNAs, where miR827 targeted both SPX-MFS proteins, depending on the approach we used (Fig. 3A). In addition, the 18 nt sequence 5'-

AATCGTCTTTACATCGGATG-3' $(3.24$, id = 2117) was found to target mRNA encoding glutaminyl-peptide cyclotransferase (HORVU7Hr1G003920, PAREsnip2: score = 2.0, MFE = -19.6) (Fig. 4C), which may be involved in plant defense reactions [101].

Some other interesting Pi-related targets which are recognized by DESs were found in our root degradome data, but the prediction scores were weaker than those in the examples described above. For instance: nitrate reductase (HORVU6Hr1G003300), high-affinity nitrate transporter-activating protein (HORVU5Hr1G115500), MYB-like TF (HORVU7Hr1G027370), and stress-induced TF NAC1 (HORVU5Hr1G111590) were found. Interestingly, among the 98 DEGs identified in this study, only two of 
them (SPX-MFS1 and SPX-MFS2) were found as putative targets of miRNA guided activity. In addition, none of the DEGs gene IDs were found to match with any of the identified IDs classified for differentially expressed small RNAs. All predicted DESs targets are listed in Additional files 14, 16, 19, and 23.

\section{Discussion}

In this study, we used a tripartite approach (sRNA-Seq, mRNA RNA-Seq, and degradome-seq) to describe the set of small RNAs differentially expressed in barley roots and shoots under low-Pi stress. We detailed the sophisticated responses of barley shoots and roots involved in the maintaining of Pi homeostasis (Fig. 6). Integrated deep-sequencing data were used to describe organ-specific adaptations to low-Pi through either activation or repression of different classes of 18-25 nt small RNAs. Additionally, the mRNA-Seq analysis of low-Pi treated barley shoot was performed to analyze the correlation between shoot-derived small RNAs, annotated to either protein-coding mRNAs (47.87\%) or non-translating RNAs (36.49\%), and gene expression changes of polyadenylated RNAs. We identified a total of 28 differentially expressed miRNAs (Bonferroni tested $p$-value) annotated to miRBase (release 22) without mismatches and a total of 1989 differentially expressed other small RNAs (Bonferroni tested $p$-value).

In plants, a limited number of miRNA have been shown to be specifically and strongly induced by $\mathrm{Pi}$ limitation, including miRNA399 [102], miRNA778 [59], miRNA827 [55], and miRNA2111 [55, 103]. In this work, the majority of DEMs represent various miR399 and miR827 isomiRs in both tested organs. Our results are consistent with sRNA sequencing data published for Arabidopsis [42, 55] and Nicotiana benthamiana L. [93]. In both plant species, authors have shown that the number of various miR399 isomiRs was the most abundant in shoots and roots under low-Pi. Eight of the 15 DEMs (after Bonferroni $p$-value correction) we found in barley shoots belonged to the miR399 family. However, in root, miR399 was represented only by one DEM; the miR399b isomiR (Fig. 3A, right panel). Previously, our absolute copy number analysis of mature miR399 demonstrated that its normalized expression level is 4-fold downregulated in barley roots, as compared to in shoots, under a low-Pi regime [104]. The long-distance movement of signal molecules is known to be crucial for Pi recycling and allocation from root to shoot. The root system is responsible for $\mathrm{Pi}$ acquisition conducted by phosphate transporters belonging to PHT1 family, which saturate cell membranes during Pi deficiency [105]. The level of PHT1 proteins is negatively controlled by the PHO2, which is suppressed by miR399 (see model in Fig. 7) [106]. A high level of miR399 molecules was detected in Arabidopsis wild type rootstocks grafted with miR399-overexpressing scions $[42,107]$. Thus, miR399 is involved in a plant's systemic response to low-Pi conditions and acts as a long-distance signal, moving from shoot to root to control Pi homeostasis [107]. In Arabidopsis, miR827 has been shown in multiple studies to target the 5'-UTR of the NITROGEN LIMITATION ADAPTATION $(N L A)$ gene $[108,109]$. In rice, the OsNLA mRNA has a 'degenerate' osa-miR827 potential cleavage site, that is why miR827 does not cleave the OsNLA transcript in vivo $[56,57,110]$. Likewise, we did not find NLA mRNAs to be targeted by any of the identified hvu-miR827 isomiRs in our barley degradome records. The NLA gene encodes an E3 ubiquitin-protein ligase with RING and SPX domains, which interacts with the $\mathrm{PHO} 2$ to prevent the excessive accumulation of Pi [111]. In roots, a more diverse set of miRNAs contributed to the compensation of low-Pi stress, compared to that in shoots. We found six up-regulated 
miRNA molecules (DEMs) in roots mapped to pre-miRNAs, such as: miR9779, miR5083, miR9779, miR5083, miR1511 and miR5072. In addition, none of them was found in our degradome analysis. The differentially expressed other small RNAs in roots (DESs, 1796 molecules) were represented by $90 \%$ of the total set of other sRNAs (DESs from both organs), annotated to all classes of cDNAs taken for analysis. Among the identified set of DESs, many putative miRNA-like molecules were predicted to target various mRNAs involved in plant adaptations to abiotic stresses, plant defense, and/or transcription (Table 1). Further analysis will be performed to experimentally validate the in silico predicted PTGS role of Pi-responsive small RNAs found in this study, as well.

In this paper, we detailed the particular shoot differentially expressed genes (DEGs) harboring Piresponsive cis-regulatory elements, involving various molecular pathways and biological processes. These DEGs were mostly engaged in Pi mobilization and utilization upon Pi-starvation in barley shoots. Other sRNAs selected from shoots were much less abundant and represent sequences belonging mostly to non-translating and/or protein-coding mRNAs. None of the sRNAs mapped to the differentially expressed mRNAs found in the transcriptomic analysis, suggesting that they may inhibit gene expression through translational repression or may serve as a Pi source for developing plant organs. Plants are adapted to recycle nutrients from senescing organs. For example, class II RNases are involved in the degradation of housekeeping rRNAs before cell death occurs [112]. During senescence extracellular class I RNases were shown to degrade RNA during Pi-starvation in Arabidopsis as well [113]. In 2018, Ren et al. published RNA-Seq data describing the barley transcriptome under low-Pi stress [114]. The authors compared the transcriptomes of two barley genotypes with contrasting low-Pi stress tolerance. In roots, they observed 28 DEGs classified into the following functional groups: Pi transport, transcription, lipid metabolism, metabolism, and phosphorylation/dephosphorylation [114]. Likewise, our RNA-Seq data from barley shoot discovered the DEGs involved in all mentioned functional groups. In our shoot transcriptome analysis, we found the same four DEGs: (i) $\underline{G} L Y C E R O P H O S P H O \underline{D} I E S T E R$ $\underline{P H O S P H O D I E S T E R A S E} 1$ (GDPD1) gene (HORVU3Hr1G079900, $\left.\log _{2}(\mathrm{fc})=5.78\right)$, (ii) MONOGALACTOSYLDIACYLGLYCEROL SYNTHASE 2 gene (MGD2, HORVU4Hr1G044140, 5.27), (iii) SPX5 (HORVU2Hr1G031400, 3.44), and (iv) SPX1 (HORVU7Hr1G089910, 1.78). Furthermore, Ren et al. found three genes encoding purple acid phosphatases (PAPs) [114]; however, they appeared from different barley genome loci than the five PAPs we found in our study. It was shown that vacuolar and secreted PAPs are involved in Pi scavenging and remobilization during Pi-starvation and leaf senescence. Other related RNA-Seq data published for either wheat (Triticum aestivum L.) [115], rice [116], soybean [117], Plantago major L. [118], and maize [119] demonstrated similar molecular patterns to those in our study (Additional file 8). Based on our work we propose a model of barley adaptations to Pi-starvation (Fig.7). Interestingly, the presence of crucial Pi-responsive cis-regulatory elements within the promoter regions of more than $50 \%$ of identified DEGs may indicate their essential and direct role in conditioning low-Pi tolerance (Fig. 6B). The most widely studied PHR TFs, such as PHR1 in Arabidopsis [82] and PHR2 in rice [120], bind to P1BS elements present in the promoter of a broad range of Pi-related genes. Moreover, the PHR protein family exhibits high functional redundancy and its protein members may co-operatively form a regulatory network to maintain Pi homeostasis in plants [84]. In our previous paper, we showed that, 
within the 5'-UTR of the $\mathrm{PHO} 2$ gene, there is another Pi-responsive motif called the $\mathrm{PHO}$ element in close proximity to the P1BS [48]. The PHO element can be bound by PHR-like transcription factors in barley plants, as well [48], and has been found in the promoters of many DEGs in independent Arabidopsis [121, 122] or soybean [117] studies.

The elevated abundance of sRNAs has been associated with the up-regulation of two types of nucleases (endonuclease S1/P1 and 3'-5' exonuclease), which may catalyze the degradation of RNA into shorter fragments $[123,124]$ and play a relevant role in nutrient mobilization under Pi-starvation. It seems likely that sRNA production upon Pi-starvation is an effect of RNA degradation by different types of nucleases. Thus, degraded RNA may serve as a source of Pi necessary in emerging plant organs. We found also two genes encoding oxalate oxidases, the expression of which was significantly downregulated during Pistarvation. This class of genes is responsible for the inactivation of oxalic acid, which mediates fungalplant pathogenesis in barley [125].

\section{Conclusion}

To conclude, our studies provide comprehensive data sets, which may serve as a rich platform for the characterization of barley responses to Pi-starvation at an RNA level. Furthermore, our data may be used as a reference tool for parallel studies in other crop plants.

\section{Materials And Methods}

\section{Plant material}

Three biological replicates of barley root and shoot samples were analyzed. One replicate consisted of three plants growing in a single pot containing $1.5 \mathrm{~kg}$ of soil mixed with sand in a 7:2 ratio. Material was collected from the barley line Rolap (obtained from the Institute of Plant Genetics of the Polish Academy of Sciences, Poznań, Poland [126]) growing under low-Pi (8 mg P/kg soil) and Pi-sufficient conditions (after addition of $60 \mathrm{mg} \mathrm{P} / \mathrm{kg}$ soil), as described before [48]. On the $23^{\text {rd }}$ day after sowing plant shoots (Zadoks decimal code 22-23 [127]), they were cut off and fresh tissue weight was measured.

Immediately afterwards, shoots and roots were collected and frozen in liquid nitrogen to be kept at $-80^{\circ} \mathrm{C}$ until use.

\section{Pi concentration measurements}

Measurements of inorganic phosphate level were performed according to the protocol we have described before [46]. The samples were measured in two technical and three biological replicates using an Infinite F200 Pro (TECAN, Switzerland).

\section{RNA isolation}


Four procedures of RNA isolation were used, depending on the following experiments: (i) small RNA expression level analysis (ddPCR using TaqMan ${ }^{\mathrm{TM}}$ MicroRNA assays, NGS of small RNAs, Northern hybridization); (ii) transcriptome analysis; (iii) degradome - PARE (Parallel Analysis of RNA Ends) [128] analysis for mRNA cleaved by miRNA; or (iv) validation of RNA-Seq data using ddPCR.

(i) Small RNA expression level analysis

RNA isolation was performed using a modified method allowing enrichment of small RNAs, according to the detailed protocol we published before [64].

(ii) RNA for RNA-Seq

RNA was extracted from a $100 \mathrm{mg}$ sample using RNA extraction buffer [104] and a Direct-Zol RNA MiniPrep Kit (Zymo Research). According to the Lexogen's SENSE mRNA-Seq Library prep kit v2 user guide DNase treatment step was omitted to avoid RNA hydrolysis.

(iii) RNA for degradome analysis

Procedure of RNA isolation from barley root and shoot (growing in low-Pi conditions) used for degradome profiling was performed using a method described by German et al. using RNA extraction buffer [128], along with some modifications that we have described previously $[64,129]$.

(iv) mRNA-Seq data validation

To validate the transcript level of significantly changed genes, we used precise dd-PCR analysis. To isolate RNA for these analyses, we used a Direct-Zol RNA MiniPrep Kit (Zymo Research) with some modifications that we have described in detail previously [104]. The RNA material was treated using DNase I enzyme from the above kit (Zymo Research).

\section{Preparation of NGS libraries}

We prepared three different NGS libraries: (i) small RNA, (ii) transcriptome - mRNA, and (iii) degradome.

\section{(i) Small RNA libraries}

Small RNA libraries were prepared using a TruSeq Small RNA Library Prep Kit (Illumina). In brief, small RNAs of $15-30$ nt in length were separated on denaturing $8 \mathrm{M}$ urea $15 \%$ polyacrylamide (PA) gel and purified and ligated to $3^{\prime}$ and $5^{\prime}$ RNA adapters. Next, the RNA fragments were reverse transcribed to run PCR: PCR products were indexed by utilization of specific RNA PCR Index Primers and PCR profile, according to the Illumina protocol (RPI, Illumina). PCR products were separated on $7 \%$ PA gel containing $1 \%$ glycerol. After $10^{\prime}$ staining by SYBR ${ }^{\text {TM }}$ Gold Nucleic Acid Gel Stain (Invitrogen, Thermo Fisher Scientific)/0.5xTBE buffer, DNA fragments of 140-160 bp in length were cut and eluted using $400 \mathrm{ml}$ elution buffer ( $50 \mathrm{mM}$ Mg-acetate, $0.5 \mathrm{M}$ ammonium acetate, $1 \mathrm{mM}$ EDTA, $0.1 \% \mathrm{SDS}$ ) after O/N incubation, $28^{\circ} \mathrm{C}, 400 \mathrm{rpm}$. Then, chloroform/phenol pH=8.0 purification libraries were precipitated using 
$1.5 \mathrm{ml}$ GlycoBlue $^{\mathrm{TM}}$ coprecipitant $(15 \mathrm{mg} / \mathrm{mL}$ ) (Ambion, Thermo Fisher Scientific) and three volumes of $100 \%$ ethanol. Purified libraries were quantified using a Qubit@ dsDNA HS Assay kit (Invitrogen, Thermo Fisher Scientific) and Qubit 3.0 Fluorometer (Invitrogen, Thermo Fisher Scientific). The quality of the libraries was analyzed using a High Sensitivity D1000 ScreenTape Assay (Agilent Technologies) and a 2200 TapeStation (Agilent Technologies). A total of 12 libraries were pooled together in equal molar ratio and sequenced by Fasteris SA (Switzerland).

\section{(ii) Degradome library construction}

Degradome library construction was performed according PARE technique described by German et al. [128]. Ligation was performed using a Rapid DNA Ligation Kit (Roche), according to the manufacturer instructions. The ligation mixture was composed of Mmel-digested PCR product and 3' DNA Adapter, kept for 6 hours at RT and at $4{ }^{\circ} \mathrm{C}$ overnight, then purified using phenol/chloroform extraction. PCR reaction was performed in a $50 \mathrm{ml}$ volume containing Mmel fragment-3'Adapter template, appropriate indexcontaining primer ( $0.5 \mathrm{mM}$ final concentration), Mmel Universal Fwd primer ( $0.5 \mathrm{mM}$ final concentration), 350 mM dNTPs, Q5 reaction buffer, and Q5® Hot Start High-Fidelity DNA Polymerase (New England Biolabs), using the following steps: $94^{\circ} \mathrm{C}$ for $2 \mathrm{~min} ; 94^{\circ} \mathrm{C}$ for $30 \mathrm{sec}, 60^{\circ} \mathrm{C}$ for $30 \mathrm{sec}, 72{ }^{\circ} \mathrm{C}$ for $30 \mathrm{sec}-$ 14 cycles; and $72{ }^{\circ} \mathrm{C}$ for 7 min. PCR products were separated on $8 \%$ PA gel containing $1 \%$ glycerol. Appropriate in length bands were cut and eluted $\mathrm{O} / \mathrm{N}$. Quantitative analysis of the purified libraries was performed using a Qubit® dsDNA HS Assay kit (Invitrogen, Thermo Fisher Scientific) and a Qubit 3.0 Fluorometer (Invitrogen, Thermo Fisher Scientific). Finally, the quality of the libraries was analyzed using a High Sensitivity D1000 ScreenTape Assay (Agilent Technologies) and a 2200 TapeStation (Agilent Technologies). Each library possessed an individual specific index. The four libraries were pooled together in equal molar ratio and sequenced by Fasteris SA (Switzerland). In present study two degradome libraries were analyzed.

\section{(iii) mRNA libraries}

Transcriptome libraries were carried out using a SENSE mRNA-Seq library prep kit v2 (Lexogen), according to the manufacturer's protocol and as previously described [48]. A $420 \mathrm{pg}$ of Spike-In RNA Variants SIRV-set3 (Lexogen) was added to $1500 \mathrm{ng}$ of total RNA. ERCC mix was used for Spike-in analysis.

\section{Library sequencing}

Sequencing of small RNAs was performed (i) internally, using a MiSeq paired-end kit to check the library quality using a MiSeq ${ }^{\circledR}$ Reagent Kit v3 (Illumina) at the Laboratory of High Throughput Technologies, Adam Mickiewicz University, Poznań, Poland. Received data showed proper library quality and low-Pi induced changes in small RNA levels. (ii) The main deep sequencing (12 small RNA libraries, degradome, mRNA) was performed externally by Fasteris SA (Switzerland).

\section{Data analysis}


Differences in small RNAs, RNAs levels, and preliminary degradome data analysis were performed using a CLC Genomics Workbench (Qiagen Aarhus A/S).

\section{Small RNA data analysis}

The trimming procedure was used with default settings for quality trimming (quality score limit 0.02 ), adapter trimming, and for removal of small RNAs longer than $25 \mathrm{nt}$ and shorter than $18 \mathrm{nt}$. Reads were extracted, counted, and normalized per 1,000,000 reads. Then, we set up the Experiment analysis for samples derived from roots and shoots separately (two-group comparison, unpaired). Empirical analysis of (EDGE) was used to find significant fold changes in small RNA expression levels between samples derived from different treated barley. Moreover, we performed EDGE Bonferroni and EDGE FDR $p$-value correction calculation. First, all small RNAs were annotated to miRBase (release 22) without mismatches and with strand-specific alignment. Then, unannotated small RNAs (i.e., those not identified in miRbase) were sorted according the lowest $p$-value. All identified differentially expressed sRNAs were annotated with internal ID numbers: IDs from 1 to 138 represent miRNAs identified in roots ( $p$-value $<0.05$ ), IDs 139-1934 represent other small RNAs identified in roots (Bonferroni $p$-value correction < 0.05), IDs 19352096 represent miRNAs identified in shoots $(p$-value $<0.05)$ and IDs represent 2097-2295 other small RNAs identified in shoots (Bonferroni $p$-value correction $<0.05$ ).

\section{Degradome data analysis}

Degradome construction was performed using two different approaches, which allowed for a more indepth analysis (Fig. 2). In the first method, the raw sequencing reads were processed by Cutadpt program (https://cutadapt.readthedocs.io/en/stable/) to trim low-quality and adapter sequences. Only sequences of length $15 \mathrm{nt}$ and above were selected for further analyses. The processed sequencing reads were aligned to the reference sequences using bowtie. The count of $5^{\prime}$-end marked cleavage sites was scored by Perl script and normalized to the depth of sequencing and total signal for each of the reference transcripts. The putative miRNA:target pairs were predicted by a custom program (targetSeek) which included the following steps: (i) calculation of perfect match MFE (minimum free energy); (ii) RNAplexbased (Vienna package) screening for sRNA:transcript pairs; (iii) filtering number of bulges and length of sequence overhangs by MFE (percent of the perfect MFE match); and (iv) calculation of prediction score using a penalty schema for loops, bulges, and G:U wobble pairing. In the second approach, we used the PAREsnip2 software [130] to generate t-plots in conjunction with five databases (Fig. 2). Potential miRNA targets are classified into one of five categories, where category 0 indicates the best miRNA-target match. The lower the alignment score, the better the alignment between the sRNA and the target site [130]. During PAREsnip2 analysis, we set the Fahlgren and Carrington targeting rules to permit a mismatch or G:U wobble at position 10 [131].

\section{Identification of DEGs}

Experiments were performed in three biological replicates of plants grown under low-Pi and control conditions. Paired-end sequencing reactions of the $150 \mathrm{nt}$ reads were performed using an Illumina 
System. Total read numbers from six samples were mapped to the barley reference genome from Ensembl Plants Genes 42 (Hordeum vulgare IBSC v2). The library's quality and sequencing accuracy were verified carefully (i) by adding Spike-in RNA Variant Control Mixes (Lexogen) (Additional file 24) and (ii) by quality trimming. RNA-Seq analysis was performed using following normalization method - TPM expression values. TPM (Transcripts Per Million) is computed with the following equation TPM . RPKM (Reads Per Kilobase of exon model per Million mapped reads) is computed using following equation RPKM . Differentially expressed genes (DEGs) were selected based on fold change calculations $(p<$ 0.05 ) and the selective Bonferroni test. RNA-Seq reads were mapped to the full-length transcripts using the CLC Genomics Workbench (QIAGEN) software, as previously described [48]. Among potential differentially expressed transcripts only those which went through restricted Bonferroni $p$-value correction were considered as differentially expressed genes (DEGs), which are listed in Additional file 8.

\section{GO analysis}

Gene ontology (GO) analyses were performed using the PANTHER in silico tool [132]. The overrepresentation binomial tests classified DEGs within GO domains (cellular component, biological process, and molecular function) with Bonferroni-corrected $p$-value $<0.05$.

\section{cis-regulatory motif localization within DEG promoters}

To analyze the enrichment of Pi-related cis-regulatory motifs, we extracted 2000 bps upstream of transcription start site from each identified DEG. Such data were directly screened to look for any either P1BS- or P-responsive PHO element consensus sequences using multiple promoter analysis with the PlantPAN3.0 tool (Chow et al. 2019).

\section{ddPCR}

To determine the absolute copy number of genes encoding IPS1, SPX-MFS1, endonuclease S1/P1, 3'-5' exonuclease, oxalate oxidase, and oxalate oxidase 2, we performed ddPCR using either EvaGreen Supermix (Bio-Rad) or TaqMan Assay (Bio-Rad) for mature miR827, according to the protocols previously described [48]. To normalize the copy number of miR827, we ran ddPCR for the ARF1 reference gene using the TaqMan Assay id: AIMSIL4 (Thermo Fisher Scientific). Absolute gene expression was shown as normalized copy number per 1000 copies of the barley ARF1 reference gene. All specific primers and probes (mature miR827, U6 snRNA) used in this paper are listed in Additional file 25.

\section{Northern blot of mature miR827}

To determine the mature miR827 expression level, we performed northern blot hybridization using a specific probe for analysis. All steps of these experiments were done according to a detailed protocol as described previously [64]. $10 \mu \mathrm{g}$ of each RNA sample was run alongside a radioactively labelled Decade Marker (Invitrogen, Thermo Scientific) on a 15\% polyacrylamide gel with 8M urea. The miR827and U6 probe sequences are available in Additional file 25. The Decade Marker (Ambion) was loaded to control 
the length of the tested RNAs. To calculate band intensity, we used the ImageQuant TL 8.1 software (GE Healthcare Life Sciences).

\section{RNA-Seq data}

Presented RNA-Seq data were submitted to GEO (NCBI) with the following accession number GSE145427, sRNA-Seq - GSE145425, transcriptome mRNA-Seq - GSE145426, degradome RNA-Seq - GSE145423.

\section{Abbreviations}

sRNA: small RNA; siRNA: small interfering RNA; Pi: inorganic phosphate; NGS: Next-Generation Sequencing; RNA-Seq: RNA Sequencing; DEM: differentially expressed miRNA; DES: differentially expressed small RNA; DEG: differentially expressed gene; GO: gene ontology; miRBase: the microRNA database; BLAST: Basic Local Alignment Search Tool; ddPCR: droplet digital Polymerase Chain Reaction; P1BS: PHR1 binding sequence; TF: transcription factor; PARE: Parallel Analysis of RNA Ends; EDGE: Empirical analysis of Differential Gene Expression; FDR: false discovery rate; MFE: minimum free energy; TPM: transcripts per million; RPKM: reads per kilo base of exon model per million mapped reads

\section{Declarations}

\section{Ethics approval and consent to participate}

Not applicable.

\section{Consent for publication}

Not applicable.

\section{Availability of data and materials}

The datasets generated and/or analyzed during the current study have been submitted to GEO database (accession numbers: GSE145423, GSE145425, GSE145426, GSE145427).

\section{Competing interests}

The authors declare that they have no competing interests.

\section{Funding}

This work was funded by the National Science Centre, Poland, on the basis of DEC2013/11/B/NZ9/01761, UMO-2016/23/B/NZ9/00857, and UMO-2015/19/N/NZ9/00218.

\section{Authors' contributions}


Conceptualization, writing - original draft preparation, writing - original draft editing, designing and preparing figures, plant material generation, Pi concentration measurements, ddPCR analysis, NGS data analysis, promoters' analysis, funding acquisition: P.S.; conceptualization, supervision and writing original draft preparation, writing - original draft editing, NGS libraries preparation, NGS data analysis, degradome profiling, ddPCR analysis, Northern-blot analysis, funding acquisition: A.P.; NGS data analysis, visualization, preparing figures: D.B.; NGS libraries preparation K.K.; NGS libraries preparation P.N., degradome profiling W.K.; original draft review Z.S.K.

\section{Acknowledgements}

The authors wish to thank Dr. Halina Pietrykowska (Adam Mickiewicz University, Poznań, Poland) for help with the degradome libraries preparation and Piotr Kopec (Adam Mickiewicz University, Poznań, Poland) for help in cDNA data preparation. MiSeq paired-read of small RNA deep sequencing (three replicas) was performed at the Laboratory of High-throughput Technologies (IBMiB, Faculty of Biology at the Adam Mickiewicz University, Poznań) funded by National Multidisciplinary Laboratory of Functional Nanomaterials NanoFun nr POIG.02.02.00-00-025/09 (Innovative Economy Operational Programme, Priority Axis 2: R\&D Infrastructure, Action 2.2: Support of Formation of Common Research Infrastructure of Scientific Units).

\section{Accession Numbers}

Sequence data from this article can be found in the Ensembl Plants database for the barley genome: IPS1 (HORVU4Hr1G079600), SPX-MFS1 (HORVU2Hr1G094690), SPX-MFS2 (HORVU6Hr1G065710), GAMyb transcription factor (HORVU3Hr1G079490), PCF6 transcription factor (HORVU2Hr1G060120), PHO2 (HORVU1Hr1G085570), ARF1 (HORVU3Hr1G079700), 3'-5' exonuclease (HORVU6Hr1G007360), S1/P1 endonuclease (HORVU2Hr1G112830), oxalate oxidase (HORVU4Hr1G005440), oxalate oxidase 2 (HORVU4Hr1G005380).

\section{References}

1. International Barley Genome Sequencing Consortium, Mayer KF, Waugh R, Brown JW, Schulman A, Langridge $P$, et al. A physical, genetic and functional sequence assembly of the barley genome. Nature. 2012;491(7426):711-6.

2. Jo W-S, Kim H-Y, Kim K-M. Development and characterization of polymorphic EST based SSR markers in barley (Hordeum vulgare). 3 Biotech. 2017;7(4):265.

3. Harwood WA. An introduction to barley: the crop and the model. Methods Mol Biol. 2019;1900:1-5.

4. Saisho D, Takeda K. Barley: Emergence as a new research material of crop science. Plant Cell Physiol. 2011;52(5):724-7. 
5. Phillips JR, Dalmay T, Bartels D. The role of small RNAs in abiotic stress. FEBS Lett. 2007;581(19):3592-7.

6. Sunkar R, Chinnusamy V, Zhu J, Zhu JK. Small RNAs as big players in plant abiotic stress responses and nutrient deprivation. Trends Plant Sci. 2007;12(7):301-9.

7. Banerjee S, Sirohi A, Ansari AA, Gill SS. Role of small RNAs in abiotic stress responses in plants. Plant Gene. 2017;11:180-9.

8. Großhans H, Filipowicz W. Molecular biology: the expanding world of small RNAs. Nature. 2008;451(7177):414-6.

9. Guleria P, Mahajan M, Bhardwaj J, Yadav SK. Plant small RNAs: biogenesis, mode of action and their roles in abiotic stresses. Genomics Proteomics Bioinformatics. 2011;9(6):183-99.

10. Borges F, Martienssen RA. The expanding world of small RNAs in plants. Nat Rev Mol Cell Biol. 2015;16(12):727-41.

11. Zhang $\mathrm{H}$, Xia R, Meyers BC, Walbot V. Evolution, functions, and mysteries of plant ARGONAUTE proteins. Curr Opin Plant Biol. 2015;27:84-90.

12. Bhattacharjee $S$, Roche $B$, Martienssen RA. RNA-induced initiation of transcriptional silencing (RITS) complex structure and function. RNA Biol. 2019;16(9):1133-46.

13. Ren B, Wang X, Duan J, Ma J. Rhizobial tRNA-derived small RNAs are signal molecules regulating plant nodulation. Science. 2019;365(6456):919-22.

14. Khraiwesh B, Zhu J-K, Zhu J. Role of miRNAs and siRNAs in biotic and abiotic stress responses of plants. Biochim Biophys Acta. 2012;1819(2):137-48.

15. Jacobs TB, Lawler NJ, LaFayette PR, Vodkin LO, Parrott WA. Simple gene silencing using the transacting siRNA pathway. Plant Biotechnol J. 2016;14(1):117-27.

16. Komiya R. Biogenesis of diverse plant phasiRNAs involves an miRNA-trigger and Dicer-processing. J Plant Res. 2017;130(1):17-23.

17. Xie Z, Allen E, Fahlgren N, Calamar A, Givan SA, Carrington JC. Expression of Arabidopsis MIRNA Genes. Plant Physiol. 2005;138(4):2145-54.

18. Kurihara Y, Watanabe Y. Arabidopsis micro-RNA biogenesis through Dicer-like 1 protein functions. Proc Natl Acad Sci USA. 2004;101(34):12753-8.

19. Budak H, Akpinar BA. Plant miRNAs: biogenesis, organization and origins. Funct Integr Genomics. 2015;15(5):523-31.

20. Fang X, Qi Y. RNAi in plants: An Argonaute-centered view. Plant Cell. 2016;28(2):272-85.

21. Valencia-Sanchez MA, Liu J, Hannon GJ, Parker R. Control of translation and mRNA degradation by miRNAs and siRNAs. Genes Dev. 2006;20(5):515-24.

22. Barciszewska-Pacak M, Milanowska K, Knop K, Bielewicz D, Nuc P, Plewka P, et al. Arabidopsis microRNA expression regulation in a wide range of abiotic stress responses. Front Plant Sci. $2015 ; 6: 410$. 
23. Meister G, Tuschl T. Mechanisms of gene silencing by double-stranded RNA. Nature. 2004;431(7006):343-9.

24. Megel C, Hummel G, Lalande S, Ubrig E, Cognat V, Morelle G, et al. Plant RNases T2, but not Dicer-like proteins, are major players of tRNA-derived fragments biogenesis. Nucleic Acids Res. 2019;47(2):941-52.

25. Martinez G, Choudury SG, Slotkin RK. tRNA-derived small RNAs target transposable element transcripts. Nucleic Acids Res. 2017;45(9):5142-52.

26. Palatnik JF, Allen E, Wu X, Schommer C, Schwab R, Carrington JC, et al. Control of leaf morphogenesis by microRNAs. Nature. 2003;425(6955):257-63.

27. Doench JG, Petersen CP, Sharp PA. siRNAs can function as miRNAs. Genes Dev. 2003;17(4):438-42.

28. Borsani O, Zhu J, Verslues PE, Sunkar R, Zhu J-K. Endogenous siRNAs derived from a pair of natural cis-antisense transcripts regulate salt tolerance in Arabidopsis. Cell. 2005;123(7):1279-91.

29. Li J, Zheng H, Zhang C, Han K, Wang S, Peng J, et al. Different virus-derived siRNAs profiles between leaves and fruits in cucumber green mottle mosaic virus-infected Lagenaria siceraria plants. Front Microbiol. 2016;7:1797.

30. Li J, Andika IB, Shen J, Lv Y, Ji Y, Sun L, et al. Characterization of rice black-streaked dwarf virus- and rice stripe virus-derived siRNAs in singly and doubly infected insect vector Laodelphax striatellus. PLoS One. 2013;8(6):e66007.

31. Zhang B, Li W, Zhang J, Wang L, Wu J. Roles of small RNAs in virus-plant interactions. Viruses. 2019;11(9):e827.

32. Kruszka K, Pacak A, Swida-Barteczka A, Nuc P, Alaba S, Wroblewska Z, et al. Transcriptionally and post-transcriptionally regulated microRNAs in heat stress response in barley. J Exp Bot. 2014;65(20):6123-35.

33. Zhou H, Hussain SS, Hackenberg M, Bazanova N, Eini O, Li J, et al. Identification and characterisation of a previously unknown drought tolerance-associated microRNA in barley. Plant J. 2018;95(1):13849.

34. Zeng J, Ye Z, He X, Zhang G. Identification of microRNAs and their targets responding to lowpotassium stress in two barley genotypes differing in low-K tolerance. J Plant Physiol. 2019;234235:44-53.

35. Wu L, Yu J, Shen Q, Huang L, Wu D, Zhang G. Identification of microRNAs in response to aluminum stress in the roots of Tibetan wild barley and cultivated barley. BMC Genomics. 2018;19(1):560.

36. Hackenberg M, Shi B, Gustafson P, Langridge P. Characterization of phosphorus-regulated miR399 and miR827 and their isomirs in barley under phosphorus-sufficient and phosphorus-deficient conditions. BMC Plant Biol. 2013;13:214.

37. Carthew RW, Sontheimer EJ. Origins and mechanisms of miRNAs and siRNAs. Cell. 2009;136(4):642-55. 
38. Fahlgren N, Montgomery TA, Howell MD, Allen E, Dvorak SK, Alexander AL, et al. Regulation of AUXIN RESPONSE FACTOR3 by TAS3 ta-siRNA affects developmental timing and patterning in Arabidopsis. Curr Biol. 2006;16(9):939-44.

39. Matsui A, Mizunashi K, Tanaka M, Kaminuma E, Nguyen AH, Nakajima M, et al. tasiRNA-ARF pathway moderates floral architecture in Arabidopsis plants subjected to drought stress. Biomed Res Int. 2014;2014:303451.

40. Dotto MC, Petsch KA, Aukerman MJ, Beatty M, Hammell M, Timmermans MC. Genome-wide analysis of leafbladeless1-regulated and phased small RNAs underscores the importance of the TAS3 tasiRNA pathway to maize development. PLoS Genet. 2014;10(12):e1004826.

41. Marin E, Jouannet V, Herz A, Lokerse AS, Weijers D, Vaucheret $H$, et al. miR390, Arabidopsis TAS3 tasiRNAs, and their AUXIN RESPONSE FACTOR targets define an autoregulatory network quantitatively regulating lateral root growth. Plant Cell. 2010;22(4):1104-17.

42. Hsieh L-C, Lin S-I, Shih A-C, Chen J-W, Lin W-Y, Tseng C-Y, et al. Uncovering small RNA-mediated responses to phosphate deficiency in Arabidopsis by deep sequencing. Plant Physiol. 2009;151(4):2120-32.

43. Abel S, Ticconi C, Delatorre CA. Phosphate sensing in higher plants. Physiol Plant. 2002;115(1):1-8.

44. Poirier Y, Bucher M. Phosphate transport and homeostasis in Arabidopsis. Arabidopsis Book. 2002;1:e0024.

45. Yang XJ, Finnegan PM. Regulation of phosphate starvation responses in higher plants. Ann Bot. 2010;105(4):513-26.

46. Pacak A, Barciszewska-Pacak M, Swida-Barteczka A, Kruszka K, Sega P, Milanowska K, et al. Heat stress affects Pi-related genes expression and inorganic phosphate deposition/accumulation in barley. Front Plant Sci. 2016;7:926.

47. Mimura T, Dietz K-J, Kaiser W, Schramm MJ, Kaiser G, Heber U. Phosphate transport across biomembranes and cytosolic phosphate homeostasis in barley leaves. Planta. 1990;180(2):139-46.

48. Sega P, Kruszka K, Szewc L, Szweykowska-Kulinska Z, Pacak A. Identification of transcription factors that bind to the 5'-UTR of the barley PHO2 gene. Plant Mol Biol. 2020;102(1-2):73-88.

49. Huang T-K, Han C-L, Lin S-I, Chen Y-J, Tsai Y-C, Chen Y-R, et al. Identification of downstream components of ubiquitin-conjugating enzyme PHOSPHATE2 by quantitative membrane proteomics in Arabidopsis roots. Plant Cell. 2013;25(10):4044-60.

50. Aung K, Lin S-I, Wu C-C, Huang Y-T, Su C-L, Chiou T-J. pho2, a phosphate overaccumulator, is caused by a nonsense mutation in a microRNA399 target gene. Plant Physiol. 2006;141(3):1000-11.

51. Chiou T-J, Aung K, Lin S-I, Wu C-C, Chiang S-F, Su C-L. Regulation of phosphate homeostasis by microRNA in Arabidopsis. Plant Cell. 2006;18(2):412-21.

52. Pacak A, Geisler K, Jørgensen B, Barciszewska-Pacak M, Nilsson L, Nielsen TH, et al. Investigations of barley stripe mosaic virus as a gene silencing vector in barley roots and in Brachypodium distachyon and oat. Plant Methods. 2010;6:26. 
53. Wang C, Ying S, Huang H, Li K, Wu P, Shou H. Involvement of OsSPX1 in phosphate homeostasis in rice. Plant J. 2009;57(5):895-904.

54. Franco-Zorrilla JM, Valli A, Todesco M, Mateos I, Puga MI, Rubio-Somoza I, et al. Target mimicry provides a new mechanism for regulation of microRNA activity. Nat Genet. 2007;39(8):1033-7.

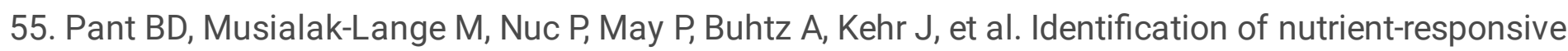
Arabidopsis and rapeseed microRNAs by comprehensive real-time polymerase chain reaction profiling and small RNA sequencing. Plant Physiol. 2009;150(3):1541-55.

56. Wang C, Huang W, Ying Y, Li S, Secco D, Tyerman S, et al. Functional characterization of the rice SPXMFS family reveals a key role of OsSPX-MFS1 in controlling phosphate homeostasis in leaves. New Phytol. 2012;196(1):139-48.

57. Lin S-I, Santi C, Jobet E, Lacut E, El Kholti N, Karlowski WM, etal. Complex regulation of two target genes encoding SPX-MFS proteins by rice miR827 in response to phosphate starvation. Plant Cell Physiol. 2010;51(12):2119-31.

58. Liu T-Y, Huang T-K, Yang S-Y, Hong Y-T, Huang S-M, Wang F-N, et al. Identification of plant vacuolar transporters mediating phosphate storage. Nat Commun. 2016;7:11095.

59. Wang L, ZengJ HQ, Song J, Feng SJ, Yang ZM. miRNA778 and SUVH6 are involved in phosphate homeostasis in Arabidopsis. Plant Sci. 2015;238:273-85.

60. Ebbs ML, Bartee L, Bender J. H3 lysine 9 methylation is maintained on a transcribed inverted repeat by combined action of SUVH6 and SUVH4 methyltransferases. Mol Cell Biol. 2005;25(23):10507-15.

61. Míguez-Montero MA, Valentine A, Pérez-Fernández MA. Regulatory effect of phosphorus and nitrogen on nodulation and plant performance of leguminous shrubs. AoB Plants. 2019; plz047.

62. Tsikou D, Yan Z, Holt DB, Abel NB, Reid DE, Madsen LH, et al. Systemic control of legume susceptibility to rhizobial infection by a mobile microRNA. Science. 2018;362(6411):233-6.

63. Kozomara A, Birgaoanu M, Griffiths-Jones S. miRBase: from microRNA sequences to function. Nucleic Acids Res. 2019;47(D1):D155-62.

64. Kruszka K, Pacak A, Swida-Barteczka A, Stefaniak AK, Kaja E, Sierocka I, et al. Developmentally regulated expression and complex processing of barley pri-microRNAs. BMC Genomics. 2013;14:34.

65. Singh A, Roy S, Singh S, Das SS, Gautam V, Yadav S, et al. Phytohormonal crosstalk modulates the expression of miR166/165s, target Class III HD-ZIPs, and KANADI genes during root growth in Arabidopsis thaliana. Sci Rep. 2017;7(1):3408.

66. Manna S. An overview of pentatricopeptide repeat proteins and their applications. Biochimie. 2015;113:93-9.

67. May A, Berger S, Hertel T, Köck M. The Arabidopsis thaliana phosphate starvation responsive gene AtPPsPase1 encodes a novel type of inorganic pyrophosphatase. Biochim Biophys Acta. 2011;1810(2):178-85.

68. Secco D, Wang C, Arpat BA, Wang Z, Poirier Y, Tyerman SD, et al. The emerging importance of the SPX domain-containing proteins in phosphate homeostasis. New Phytol. 2012;193(4):842-51. 
69. Secco D, Baumann A, Poirier Y. Characterization of the rice PHO1 gene family reveals a key role for OsPH01;2 in phosphate homeostasis and the evolution of a distinct clade in dicotyledons. Plant Physiol. 2010;152(3):1693-704.

70. Pandey BK, Mehra P, Verma L, Bhadouria J, Giri J. OsHAD1, a haloacid dehalogenase-like APase, enhances phosphate accumulation. Plant Physiol. 2017;174(4):2316-32.

71. Tran HT, Hurley BA, Plaxton WC. Feeding hungry plants: the role of purple acid phosphatases in phosphate nutrition. Plant Sci. 2010;179(1-2):14-27.

72. Heber U, Viil J, Neimanis S, Mimura T, Dietz K-J. Photoinhibitory damage to chloroplasts under phosphate deficiency and alleviation of deficiency and damage by photorespiratory reactions. Zeitschrift für Naturforsch C. 1989;44:524-36.

73. Hernández I, Munné-Bosch S. Linking phosphorus availability with photo-oxidative stress in plants. J Exp Bot. 2015;66(10):2889-900.

74. Ma T-L, Wu W-H, Wang Y. Transcriptome analysis of rice root responses to potassium deficiency. BMC Plant Biol. 2012;12:161.

75. Gho Y-S, Kim S-J, Jung K-H. Phenylalanine ammonia-lyase family is closely associated with response to phosphate deficiency in rice. Genes Genomics. 2020;42(1):67-76.

76. Thiebaut F, Rojas CA, Grativol C, Motta MR, Vieira T, Regulski M, et al. Genome-wide identification of microRNA and siRNA responsive to endophytic beneficial diazotrophic bacteria in maize. BMC Genomics. 2014;15:766.

77. Khandal H, Singh AP, Chattopadhyay D. MicroRNA397b-LACCASE2 module regulates root lignification under water- and phosphate deficiency. Plant Physiol. 2020;pp.00921.2019.

78. Maeda Y, Konishi M, Kiba T, Sakuraba Y, Sawaki N, Kurai T, et al. A NIGT1-centred transcriptional cascade regulates nitrate signalling and incorporates phosphorus starvation signals in Arabidopsis. Nat Commun. 2018;9(1):1376.

79. Medici A, Marshall-Colon A, Ronzier E, Szponarski W, Wang R, Gojon A, et al. AtNIGT1/HRS1 integrates nitrate and phosphate signals at the Arabidopsis root tip. Nat Commun. 2015;6:6274.

80. Hu B, Jiang Z, Wang W, Qiu Y, Zhang Z, Liu Y, et al. Nitrate-NRT1.1B-SPX4 cascade integrates nitrogen and phosphorus signalling networks in plants. Nat Plants. 2019;5(4):401-13.

81. Bustos R, Castrillo G, Linhares F, Puga MI, Rubio V, Pérez-Pérez J, et al. A central regulatory system largely controls transcriptional activation and repression responses to phosphate starvation in Arabidopsis. PLoS Genet. 2010;6(9):e1001102.

82. Rubio V, Linhares F, Solano R, Martín AC, Iglesias J, Leyva A, et al. A conserved MYB transcription factor involved in phosphate starvation signaling both in vascular plants and in unicellular algae. Genes Dev. 2001;15(16):2122-33.

83. Mukatira UT, Liu C, Varadarajan DK, Raghothama KG. Negative regulation of phosphate starvationinduced genes. Plant Physiol. 2001;127(4):1854-62.

84. Sega P, Pacak A. Plant PHR transcription factors: put on a map. Genes (Basel). 2019;10(12):e1018. 
85. Chow C-N, Lee T-Y, Hung Y-C, Li G-Z, Tseng K-C, Liu Y-H, et al. PlantPAN3.0: a new and updated resource for reconstructing transcriptional regulatory networks from ChIP-seq experiments in plants. Nucleic Acids Res. 2019;47(D1):D1155-63.

86. Kuo H-F, Chang T-Y, Chiang S-F, Wang W-D, Charng Y-Y, Chiou T-J. Arabidopsis inositol pentakisphosphate 2-kinase, AtIPK1, is required for growth and modulates phosphate homeostasis at the transcriptional level. Plant J. 2014;80(3):503-15.

87. Nimmo HG, Fontaine V, Hartwell J, Jenkins GI, Nimmo GA, Wilkins MB. PEP carboxylase kinase is a novel protein kinase controlled at the level of expression. New Phytol. 2001;151(1):91-7.

88. Percudani R, Peracchi A. A genomic overview of pyridoxal-phosphate-dependent enzymes. EMBO Rep. 2003;4(9):850-4.

89. Hernandez JM, Feller A, Morohashi K, Frame K, Grotewold E. The basic helix loop helix domain of maize $\mathrm{R}$ links transcriptional regulation and histone modifications by recruitment of an EMSY-related factor. Proc Natl Acad Sci USA. 2007;104(43):17222-7.

90. Brasil JN, Cabral LM, Eloy NB, Primo LM, Barroso-Neto IL, Grangeiro LP, et al. AIP1 is a novel Agenet/Tudor domain protein from Arabidopsis that interacts with regulators of DNA replication, transcription and chromatin remodeling. BMC Plant Biol. 2015;15:270.

91. Luo L, Li W, Miura K, Ashikari M, Kyozuka J. Control of tiller growth of rice by OsSPL14 and strigolactones, which work in two independent pathways. Plant Cell Physiol. 2012;53(10):1793-801.

92. Huang S, Liang Z, Chen S, Sun H, Fan X, Wang C, et al. A transcription factor, OsMADS57, regulates long-distance nitrate transport and root elongation. Plant Physiol. 2019;180(2):882-95.

93. Huen A, Bally J, Smith P. Identification and characterisation of microRNAs and their target genes in phosphate-starved Nicotiana benthamiana by small RNA deep sequencing and 5'RACE analysis. BMC Genomics. 2018;19(1):940.

94. Swida-Barteczka A, Szweykowska-Kulinska Z. Micromanagement of developmental and stressinduced senescence: the emerging role of microRNAs. Genes (Basel). 2019;10(3):e210.

95. Gubler F, Chandler PM, White RG, Llewellyn DJ, Jacobsen JV. Gibberellin signaling in barley aleurone cells. Control of SLN1 and GAMYB expression. Plant Physiol. 2002;129(1):191-200.

96. Cao D, Xu H, Zhao Y, Deng X, Liu Y, Soppe WJ, Lin J. Transcriptome and degradome sequencing reveals dormancy mechanisms of Cunninghamia lanceolata seeds. Plant Physiol. 2016;172(4):2347-62.

97. Zhao H, Wu D, Kong F, Lin K, Zhang H, Li G. The Arabidopsis thaliana nuclear factor Y transcription factors. Front Plant Sci. 2017;7:2045.

98. Zhou X, Zha M, Huang J, Li L, Imran M, Zhang C. StMYB44 negatively regulates phosphate transport by suppressing expression of PHOSPHATE1 in potato. J Exp Bot. 2017;68(5):1265-81.

99. Zhang A, Jiang X, Zhang F, Wang T, Zhang X. Dynamic response of RNA editing to temperature in grape by RNA deep sequencing. Funct Integr Genomics. 2019. [Epub ahead of print] 
100. Takenaka M, Zehrmann A, Verbitskiy D, Kugelmann M, Härtel B, Brennicke A. Multiple organellar RNA editing factor (MORF) family proteins are required for RNA editing in mitochondria and plastids of plants. Proc Natl Acad Sci U S A. 2012;109(13):5104-9.

101. Schilling S, Stenzel I, von Bohlen A, Wermann M, Schulz K, Demuth H-U, et al. Isolation and characterization of the glutaminyl cyclases from Solanum tuberosum and Arabidopsis thaliana: implications for physiological functions. Biol Chem. 2007;388(2):145-53.

102. Bari R, Pant BD, Stitt M, Scheible W-R. PHO2, microRNA399, and PHR1 define a phosphate-signaling pathway in plants. Plant Physiol. 2006;141(3):988-99.

103. Thomas HR, Frank MH. Connecting the pieces: uncovering the molecular basis for long-distance communication through plant grafting. New Phytol. 2019;223(2):582-9.

104. Smoczynska A, Sega P, Stepien A, Knop K, Jarmolowski A, Pacak A, et al. miRNA detection by stemloop RT-qPCR in studying microRNA biogenesis and microRNA responsiveness to abiotic stresses. In: de Folter S. (eds) Plant MicroRNAs. Methods Mol Biol. 2019;1932:131-50.

105. Jost R, Pharmawati M, Lapis-Gaza HR, Rossig C, Berkowitz O, Lambers H, et al. Differentiating phosphate-dependent and phosphate-independent systemic phosphate-starvation response networks in Arabidopsis thaliana through the application of phosphite. J Exp Bot. 2015;66(9):250114.

106. Kim W, Ahn HJ, Chiou T-J, Ahn JH. The role of the miR399-PHO2 module in the regulation of flowering time in response to different ambient temperatures in Arabidopsis thaliana. Mol Cells. 2011;32(1):83-8.

107. Lin S-I, Chiang S-F, Lin W-Y, Chen J-W, Tseng C-Y, Wu P-C, et al. Regulatory network of microRNA399 and $\mathrm{PHO} 2$ by systemic signaling. Plant Physiol. 2008;147(2):732-46.

108. Kant S, Peng M, Rothstein SJ. Genetic regulation by NLA and microRNA827 for maintaining nitratedependent phosphate homeostasis in Arabidopsis. PLoS Genet. 2011;7(3):e1002021.

109. Lin W-Y, Huang T-K, Chiou T-J. NITROGEN LIMITATION ADAPTATION, a target of microRNA827, mediates degradation of plasma membrane-localized phosphate transporters to maintain phosphate homeostasis in Arabidopsis. Plant Cell. 2013;25(10):4061-74.

110. Yang S-Y, Lu W-C, Ko S-S, Sun C-M, Hung J-C, Chiou T-J. Upstream open reading frame and phosphate-regulated expression of rice OsNLA1 controls phosphate transport and reproduction. Plant Physiol. 2020;182(1):393-407.

111. Park BS, Seo JS, Chua N-H. NITROGEN LIMITATION ADAPTATION recruits PHOSPHATE2 to target the phosphate transporter PT2 for degradation during the regulation of Arabidopsis phosphate homeostasis. Plant Cell. 2014;26(1):454-64.

112. Stigter KA, Plaxton WC. Molecular mechanisms of phosphorus metabolism and transport during leaf senescence. Plants. 2015;4(4):773-98.

113. Bariola PA, Maclntosh GC, Green PJ. Regulation of S-like ribonuclease levels in Arabidopsis. Antisense inhibition of RNS1 or RNS2 elevates anthocyanin accumulation. Plant Physiol. 1999;119(1):331-42. 
114. Ren P, Meng Y, Li B, Ma X, Si E, Lai Y, et al. Molecular mechanisms of acclimatization to phosphorus starvation and recovery underlying full-length transcriptome profiling in barley (Hordeum vulgare L.). Front Plant Sci. 2018;9:500.

115. Oono Y, Kobayashi F, Kawahara Y, Yazawa T, Handa H, Itoh T, et al. Characterisation of the wheat (Triticum aestivum L.) transcriptome by de novo assembly for the discovery of phosphate starvationresponsive genes: gene expression in Pi-stressed wheat. BMC Genomics. 2013;14:77.

116. Oono $Y$, Kawahara $Y$, Yazawa $T$, Kanamori $H$, Kuramata $M$, Yamagata $H$, et al. Diversity in the complexity of phosphate starvation transcriptomes among rice cultivars based on RNA-Seq profiles. Plant Mol Biol. 2013;83(6):523-37.

117. Zeng $H$, Zhang $X$, Zhang $X$, Pi E, Xiao L, Zhu Y. Early transcriptomic response to phosphate deprivation in soybean leaves as revealed by RNA-sequencing. Int J Mol Sci. 2018;19(7):pii.E2145.

118. Huang J, Huang Z, Zhou X, Xia C, Imran M, Wang S, et al. Tissue-specific transcriptomic profiling of Plantago major provides insights for the involvement of vasculature in phosphate deficiency responses. Mol Genet Genomics. 2019;294(1):159-75.

119. Du Q, Wang K, Xu C, Zou C, Xie C, Xu Y, et al. Strand-specific RNA-Seq transcriptome analysis of genotypes with and without low-phosphorus tolerance provides novel insights into phosphorus-use efficiency in maize. BMC Plant Biol. 2016;16(1):222.

120. Zhou J, Jiao F, Wu Z, Li Y, Wang X, He X, et al. OsPHR2 is involved in phosphate-starvation signaling and excessive phosphate accumulation in shoots of plants. Plant Physiol. 2008;146(4):1673-86.

121. Hammond JP, Bennett MJ, Bowen HC, Broadley MR, Eastwood DC, May ST, et al. Changes in gene expression in Arabidopsis shoots during phosphate starvation and the potential for developing smart plants. Plant Physiol. 2003;132(2):578-96.

122. Müller R, Morant M, Jarmer H, Nilsson L, Nielsen TH. Genome-wide analysis of the Arabidopsis leaf transcriptome reveals interaction of phosphate and sugar metabolism. Plant Physiol. 2007;143(1):156-71.

123. Diaz-Baena M, Galvez-Valdivieso G, Delgado-Garcia E, Pineda M, Piedras P. Nuclease and ribonuclease activities in response to salt stress: identification of PvRNS3, a T2/S-like ribonuclease induced in common bean radicles by salt stress. Plant Physiol Biochem. 2020;147:235-41.

124. Zheng J, Wang Y, He Y, Zhou J, Li Y, Liu Q, et al. Overexpression of an S-like ribonuclease gene, OsRNS4, confers enhanced tolerance to high salinity and hyposensitivity to phytochrome-mediated light signals in rice. Plant Sci. 2014;214:99-105.

125. Zhang Z, Collinge DB, Thordal-Christensen $\mathrm{H}$. Germin-like oxalate oxidase, a $\mathrm{H}_{2} \mathrm{O}_{2}$-producing enzyme, accumulates in barley attacked by the powdery mildew fungus. Plant J. 1995;8(1):139-45.

126. Devaux P, Adamski T, Surma M. Inheritance of seed set in crosses of spring barley and Hordeum bulbosum L. Crop Science. 1992;32(1):269-71.

127. Zadoks JC, Chang TT, Konzak CF. A decimal code for the growth stages of cereals. Weed Res. 1974;14(6):415-21. 
128. German MA, Luo S, Schroth G, Meyers BC, Green PJ. Construction of Parallel Analysis of RNA Ends ( PARE ) libraries for the study of cleaved miRNA targets and the RNA degradome. Nat Protoc. 2009;4(3):356-62.

129. Alaba S, Piszczalka P, Pietrykowska H, Pacak AM, Sierocka I, Nuc PW, et al. The liverwort Pellia endiviifolia shares microtranscriptomic traits that are common to green algae and land plants. New Phytol. 2015;206(1):352-67.

130. Thody J, Folkes L, Medina-Calzada Z, Xu P, Dalmay T, Moulton V. PAREsnip2: a tool for highthroughput prediction of small RNA targets from degradome sequencing data using configurable targeting rules. Nucleic Acids Res. 2018;46(17):8730-39.

131. Fahlgren N, Carrington JC. miRNA Target Prediction in Plants. In: B.C. Meyers and P.J. Green (eds.) Plant microRNA Methods. Methods Mol Biol. 2010;592(8):51-7.

132. Mi H, Muruganujan A, Ebert D, Huang X, Thomas PD. PANTHER version 14: more genomes, a new PANTHER GO-slim and improvements in enrichment analysis tools. Nucleic Acids Res. 2019;47(D1):D419-26.

133. Shi J, Dong M, Li L, Liu L, Luz-Madrigal A, Tsonis PA, et al. mirPRo-a novel standalone program for differential expression and variation analysis of miRNAs. Sci Rep. 2015;5:14617.

\section{Tables}

Due to technical limitations, full-text HTML conversion of the Tablescould not be completed. However, they can be downloaded and accessed as a PDF in the Supplementary Files.

Table 1. Degradome profiles for best scoring (score range 0-2) differentially expressed miRNAs (DEMs) identified in this study. Green color = DEMs from shoot, brown color = DEMs from root.

Table 2. List of genes predicted in degradome analysis to be guided for cleavage by the most up- and down-regulated sRNAs identified in this study.

Table 3. List of genes predicted in degradome analysis to be guided for cleavage by putative regulatory sRNAs (identified as DES) with best scoring matches.

\section{Additional Files}

Additional file 1. Normalized copy numbers of barley IPS1 gene transcript in low-Pi treated root material. DdPCR was performed to examine the absolute gene expression of the barley IPS1 gene. Obtained copy numbers were normalized per 1000 copies of the ARF1 reference gene transcript. Asterisks indicate a significant differences (* $p$-value $<0.05)$ calculated using two-tailed Student's $t$-tests.

Additional file 2. Characteristic of reads obtained from small RNA deep sequencing. 
Additional file 3. MicroRNAs and small RNAs (other) for which expression is significantly changed during Pi-starvation in barley roots and shoots. ID numbers $1-138$ : miRNAs identified in roots ( $p$-value < 0.05$)$; ID numbers 139-1934: other small RNAs identified in roots (Bonferroni $p$-value correction < 0.05); ID numbers 1935-2096: miRNAs identified in shoots ( $p$-value <0.05); ID numbers 2097-2295: other small RNAs identified in shoots (Bonferroni $p$-value correction < 0.05). Samples R4-R6 = low-Pi root; R16-R18 = control; S4-S6 = low-Pi shoot; S16-S18 = control shoot. NaN means "Not a Number", describing molecules that were exclusively expressed in low-Pi or control samples. Data created using CLC Genomics Workbench.

Additional file 4. List of differentially expressed other small RNAs (ID 2097-2295) in barley shoots (low-Pi vs. control) identified in this study (Bonferroni corrected $p$-value $<0.05$ ). Based on the available Ensembl Plants database, we classified each sequence into best-matching functional classes of cDNAs.

Additional file 5. List of differentially expressed other small RNAs (ID 139-1934) in barley roots (low-Pi vs. control) identified in this study (Bonferroni corrected $p$-value $<0.05$ ). Based on the available Ensembl Plants database, we classified each sequence into best-matching functional classes of cDNAs.

Additional file 6. Length distribution of DESs identified in barley roots and shoots.

Additional file 7. Annotation distribution of DESs identified in barley roots and shoots.

Additional file 8. List of 98 DEGs from barley shoots (low-Pi vs. control/sufficient Pi) identified in this study.

Additional file 9. Upstream sequences $(2 \mathrm{~kb})$ extracted from all 98 DEGs used for cis-regulatory motif prediction analysis.

Additional file 10. List of identified P1BS motifs within the DEG promoters.

Additional file 11. List of identified P-responsive PHO motifs within the DEG promoters.

Additional file 12. Degradome profile (TargetSeek approach) demonstrates potential mRNA targets for differentially expressed miRNAs (DEMs) identified in barley shoots (low-Pi vs. control). The lower the alignment score, the more reliable the prediction.

Additional file 13. Degradome profile (TargetSeek approach) demonstrates potential mRNA targets for differentially expressed other sRNAs (DESs) identified in barley shoots (low-Pi vs. control). The lower the alignment score, the more reliable the prediction.

Additional file 14. Degradome profile (TargetSeek approach) demonstrates potential mRNA targets for differentially expressed miRNAs (DEMs) identified in barley roots (low-Pi vs. control). The lower the alignment score, the more reliable the prediction. 
Additional file 15. Degradome profile (TargetSeek approach) demonstrates potential mRNA targets for differentially expressed other sRNAs (DESs) identified in barley roots (low-Pi vs. control). The lower the alignment score, the more reliable the prediction.

Additional file 16. Degradome profile (PAREsnip2 approach) demonstrates potential mRNA targets for differentially expressed miRNAs (DEMs) identified in barley shoots (low-Pi vs. control). The lower the alignment score, the more reliable the prediction.

Additional file 17. The t-plots generated by PAREsnip2 software showing the potential mRNA targets for differentially expressed miRNAs (DEMs) identified in barley shoots (low-Pi vs. control).

Additional file 18. Degradome profile (PAREsnip2 approach) demonstrates potential mRNA targets for differentially expressed other sRNAs (DESs) identified in barley shoots (low-Pi vs. control). The lower the alignment score, the more reliable the prediction.

Additional file 19. The t-plots generated by PAREsnip2 software showing the potential mRNA targets for differentially expressed other sRNAs (DESs) identified in barley shoots (low-Pi vs. control).

Additional file 20. Degradome profile (PAREsnip2 approach) demonstrates potential mRNA targets for differentially expressed miRNAs (DEMs) identified in barley roots (low-Pi vs. control). The lower the alignment score, the more reliable the prediction.

Additional file 21. The t-plots generated by PAREsnip2 software showing the potential mRNA targets for differentially expressed miRNAs (DEMs) identified in barley roots (low-Pi vs. control).

Additional file 22. Degradome profile (PAREsnip2 approach) demonstrates potential mRNA targets for differentially expressed other sRNAs (DESs) identified in barley roots (low-Pi vs. control). The lower the alignment score, the more reliable the prediction.

Additional file 23. The t-plots generated by PAREsnip2 software showing the potential mRNA targets for differentially expressed other sRNAs (DESs) identified in barley roots (low-Pi vs. control).

Additional file 24. Spike-in quality control of RNA-Seq samples from barley shoots (low-Pi vs. control). Correlation between known and measured spike-in concentrations.

Additional file 25. List of primers and probes used in this study.

Additional file 26. Original, full-length blot of mature hvu-miR827 analysis. Lane 1: Decade ${ }^{\text {TM Marker }}$ System (Invitrogen, Thermo Fisher Scientific); Lane 2: empty space (no sample loaded); Lane 3-5: RNA samples from root (Pi sufficient); Lane 6-8: shoot (Pi sufficient); Lane 9-11: root (low-Pi); Lane: 12-14: shoot (low-Pi).

Additional file 27. Original, full-length blot of U6 snRNA analysis. Lane 1: Decade ${ }^{\text {TM }}$ Marker System (Invitrogen, Thermo Fisher Scientific); Lane 2: empty space (no sample loaded); Lane 3-5: RNA samples 
from root (Pi sufficient); Lane 6-8: shoot (Pi sufficient); Lane 9-11: root (low-Pi); Lane: 12-14: shoot (low$\mathrm{Pi})$.

\section{Figures}

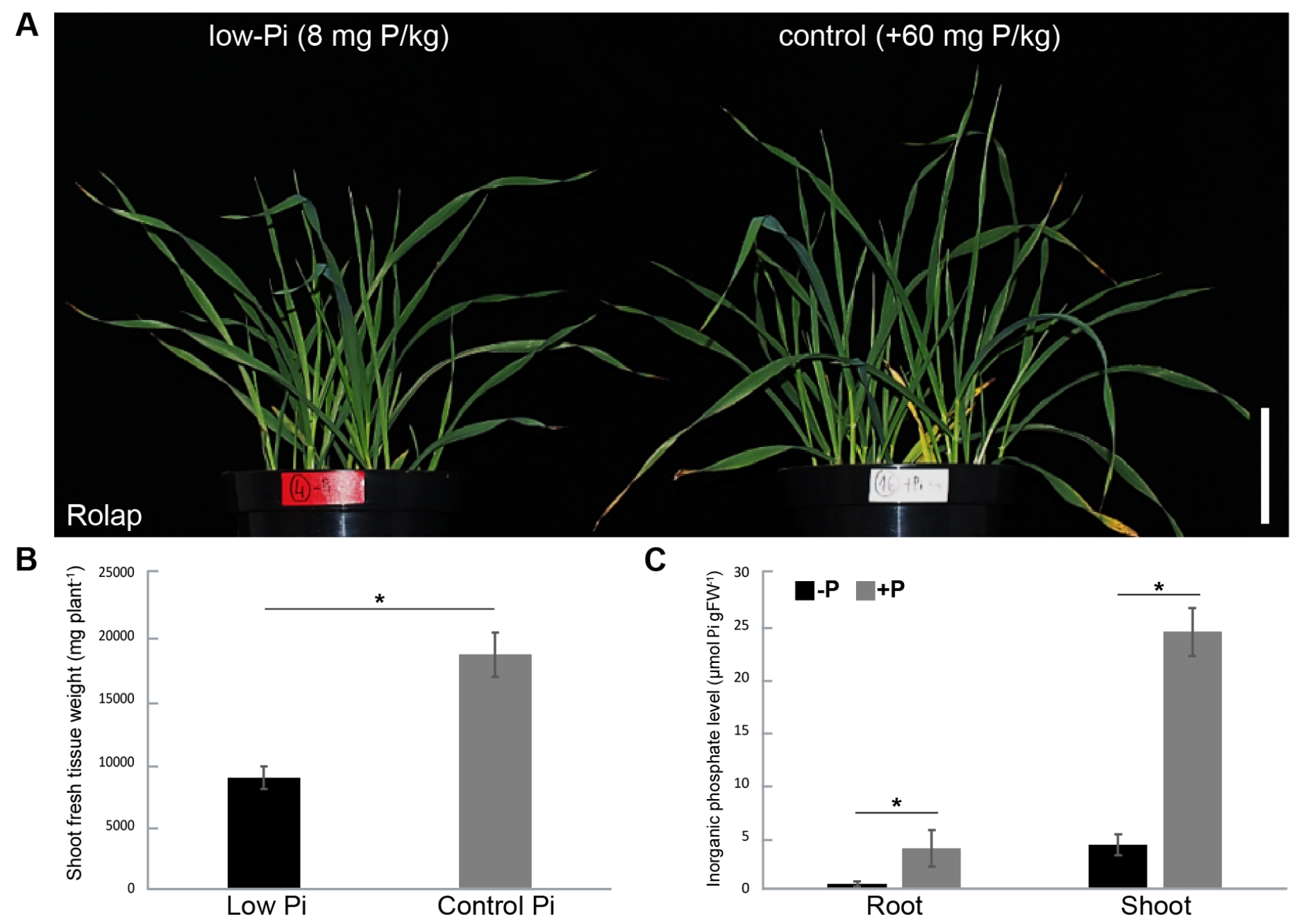

\section{Figure 1}

The validation of barley line Rolap plant material under low-Pi stress. (A) Pictures of the plants $(n=3)$ collected on the 23rd day after sowing, grown under low-Pi, $8 \mathrm{mg} \mathrm{P} / \mathrm{kg}$ soil (left) and control-Pi, addition of $60 \mathrm{mg} \mathrm{P} / \mathrm{kg}$ soil (right), conditions. (B) Shoot fresh tissue weight $(n=3)$. (C) The Pi concentration measurements performed for barley roots and shoots $(n=3)$. Asterisks indicate a significant difference $(* \mathrm{p}$-value $<0.05)$ calculated using two-tailed Student's t-tests. Scale bar $=10 \mathrm{~cm}$. Error bars $=$ SD. 

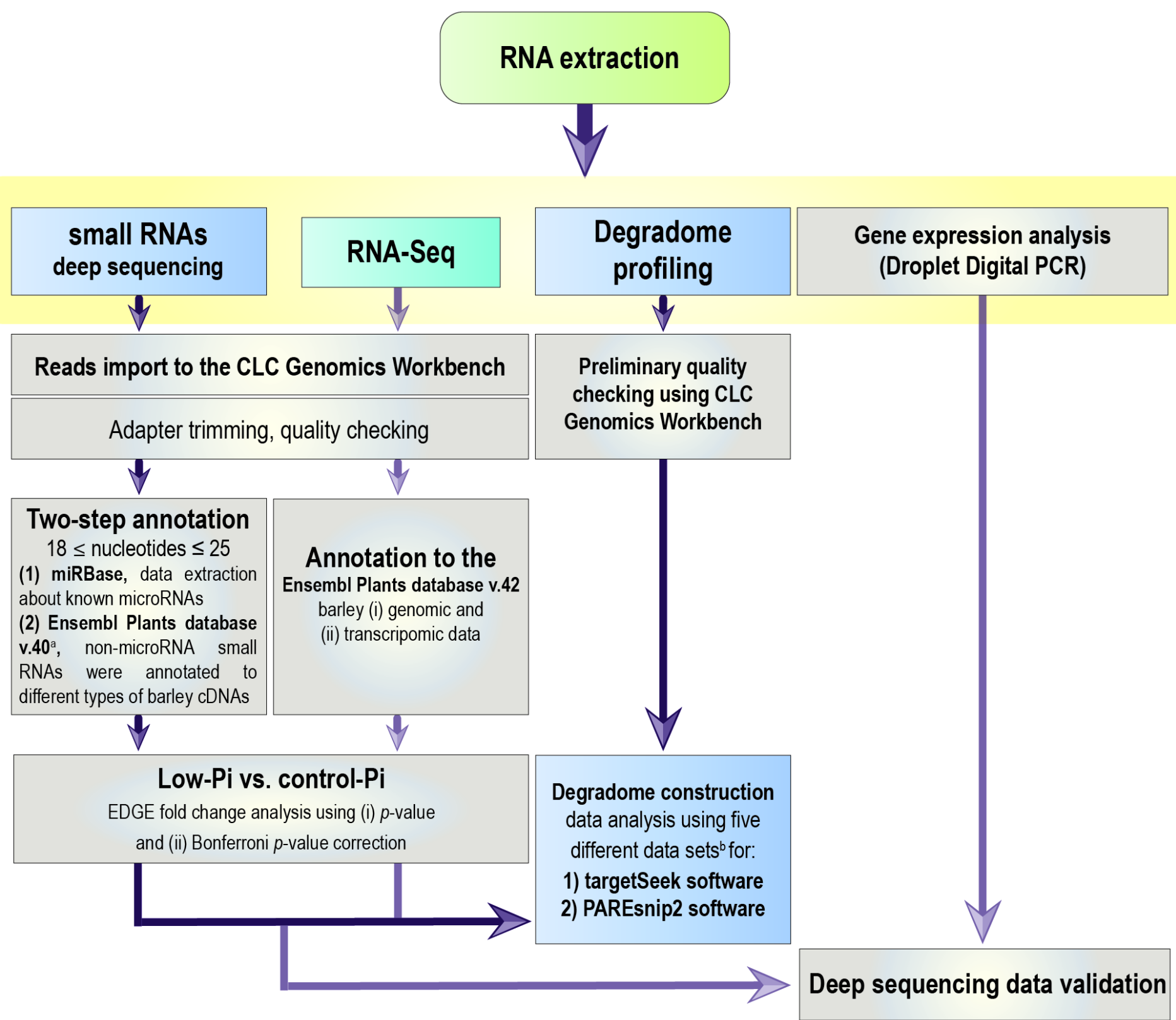

a Ensembl Plants v.40; Hordeum vulgare genes (IBSC v2), 42859 genes classified as: (i) antisense RNA - 22, (ii) nontranslating RNA - 2340 , (iii) pre-miRNA - 77, (iv) protein coding - 37 705, (v) pseudogene - 100, (vi) RNAse -1, (vii) rRNA-1 385, (viii) sense intronic - 173, (ix) snoRNA - 455, (x) snRNA - 192, (xi) SRP_RNA - 11, (xii) tRNA - 688

b (i) microRNA reads with siginicant $p$-value from roots and shoots, (ii) small RNA reads with significant (Bonferroni corrected) $p$-value from roots and shoots, (iii) protein coding cDNAs annotated to Ensembl Plants database, (iv) transcripts of 98 genes with significant expression changed upon low-Pi and (v) degradome profiles from roots and shoots

\section{Figure 2}

The framework illustrating the data generation protocols used in this study. The low-Pi stress-specific subsets of RNAs were generated following (i) deep sequencing of small RNAs from barley shoots and roots, (ii) transcriptomic RNA-Seq for barley shoots, and (iii) degradome profiling for barley shoots and roots. The obtained data sets were mapped to the references collected from miRBase and Ensembl Plants database exclusively for barley. The log2 scale for fold change and Bonferroni corrections were calculated to pick the significantly changed sequences under Pi-deficient and Pi-sufficient conditions. 
A

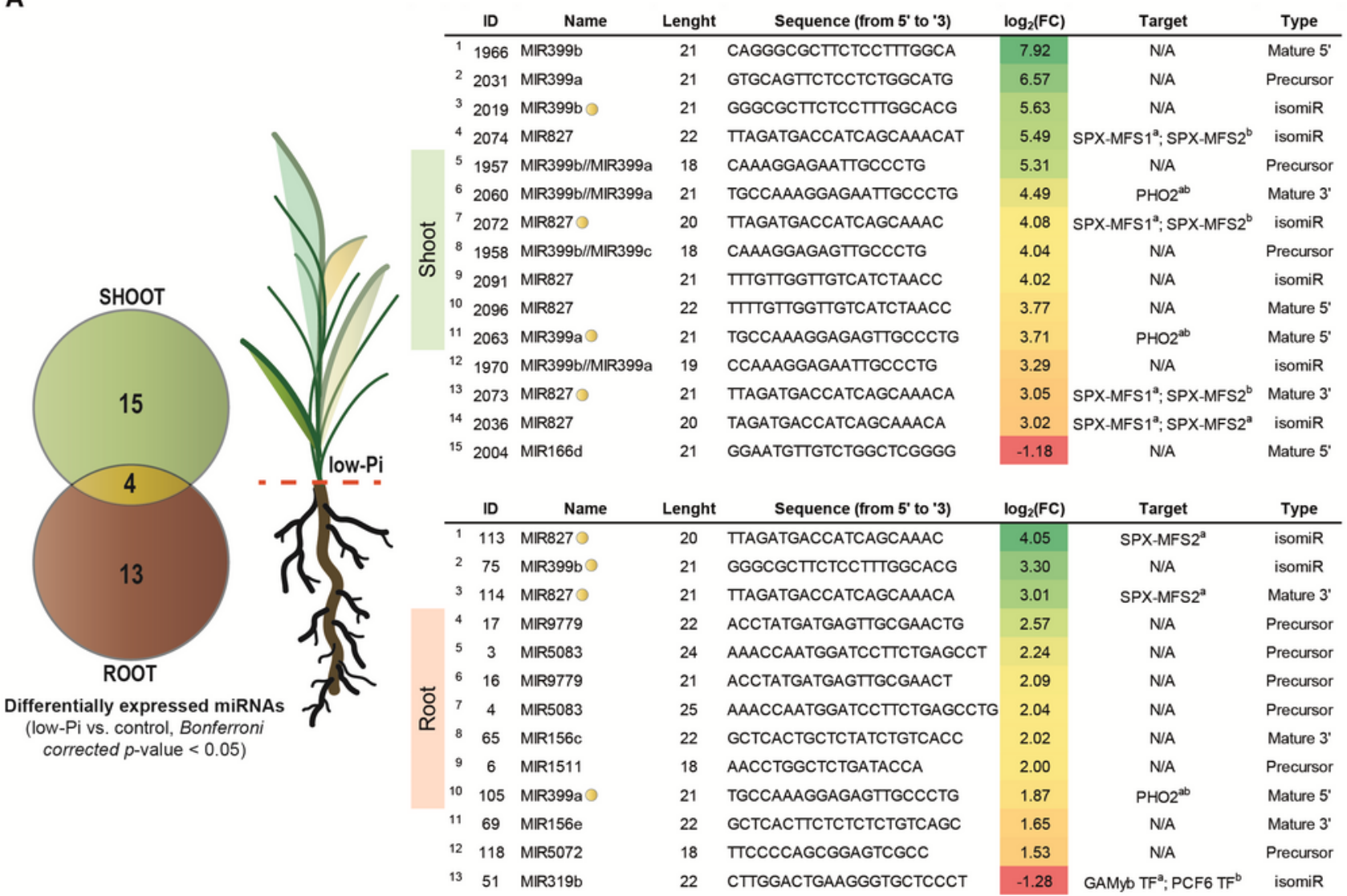

B

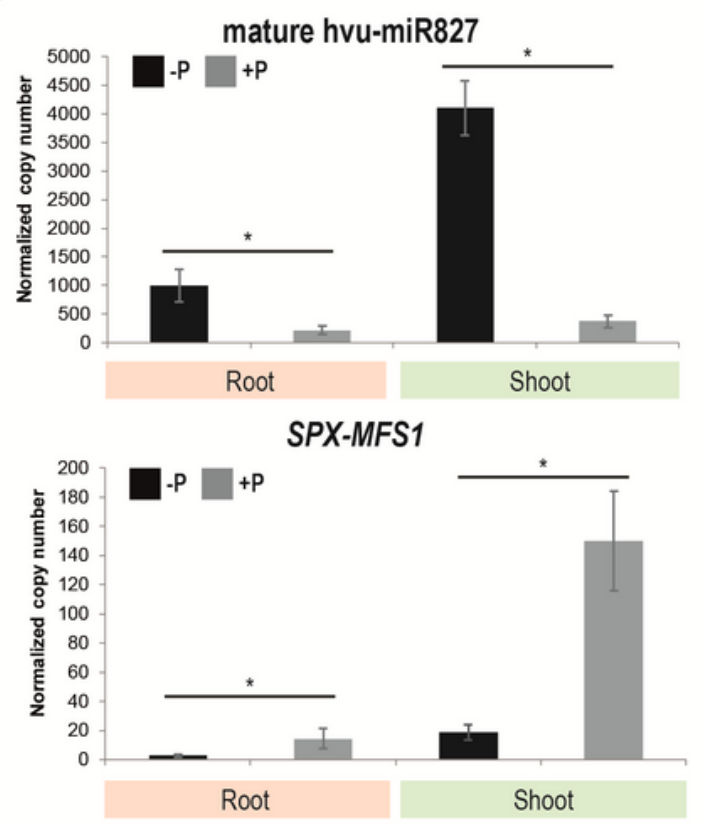

C

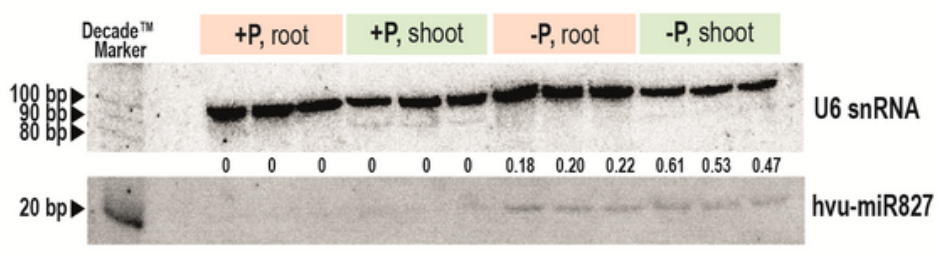

D

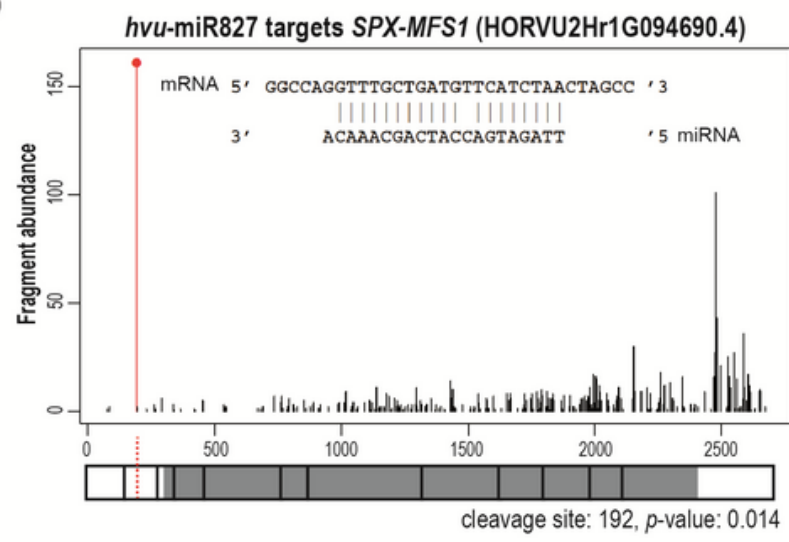

Figure 3

Differentially expressed microRNAs (DEMs) in barley plants under low-Pi regime. (left panel, A) Venn's diagram illustrating the number of identified DEMs with p-value $<0.05$ (left panel). The reduced list of DEMs that passed the Bonferroni test is presented in the right panel. The ID specifies the miRNA sequence, according to data sets obtained in NGS (Additional file 3). The given fold change is shown as a log2 value in the column log2(fc). Targeta = TargetSeek approach; Targetb = PAREsnip2 approach. 
Predicted target genes are presented in the table based on our dual degradome profiling (Additional files $13,15,17,21$ ). (right panel, A) Type categorizes miRNAs based on the sequences deposited in miRBase without mismatches, isomiRs include miRNAs with nucleotide shift (super or sub) at their $5^{\prime}, 3^{\prime}$, or at both ends [133]. Yellow dots mark miRNAs differentially expressed in both organs; shoot ID 2019 corresponds to root ID 75 and, similarly, ID $2072=$ ID 113, ID $2063=$ ID 105, and ID $2073=$ ID 114. (B) The absolute gene expression quantification of identified mature hvu-miR827 and its predicted target gene SPX-MFS1 using ddPCR. The bars represent copy numbers normalized to 1000 copies of the ARF1 reference gene; ${ }^{*}$ p-value $<0.05$, calculated using two-tailed Student's t-tests for three biological and two technical replicates. Error bars $=$ SD. (C) Detection of hvu-miR827 expression pattern in barley samples used in this study for NGS analysis. Specific probes for hvu-miR827 mature sequence and U6 reference gene were used for Northern hybridization performed on a single membrane. The number represents hvu-miR827 band intensity compared to U6 snRNA. The blots were cropped and original, full-length blots are presented in Additional file 26 and Additional file 27. (D) Degradome profile of SPX-MFS1 gene generated with DEMs from barley shoot using the PAREsnip2 approach. The red vertical line shows the cleavage position directed by hvu-miR827; the cleavage position 192 is within exon no. 2 in the 5'-UTR of the SPXMFS1 transcript ( $p$-value $=0.014$ ). The black vertical lines show the positions within the SPX-MFS1 cDNA to which degradome fragments (reads) were mapped. The number of reads (fragment abundance) is depicted by the height of the red and black lines. Below the graph, the structure of the SPX-MFS1 transcript is presented. The white boxes denote UTRs, the gray boxes denote coding sequence, and the red dotted line denote the cleavage site within the $5^{\prime}$-UTR. 
C

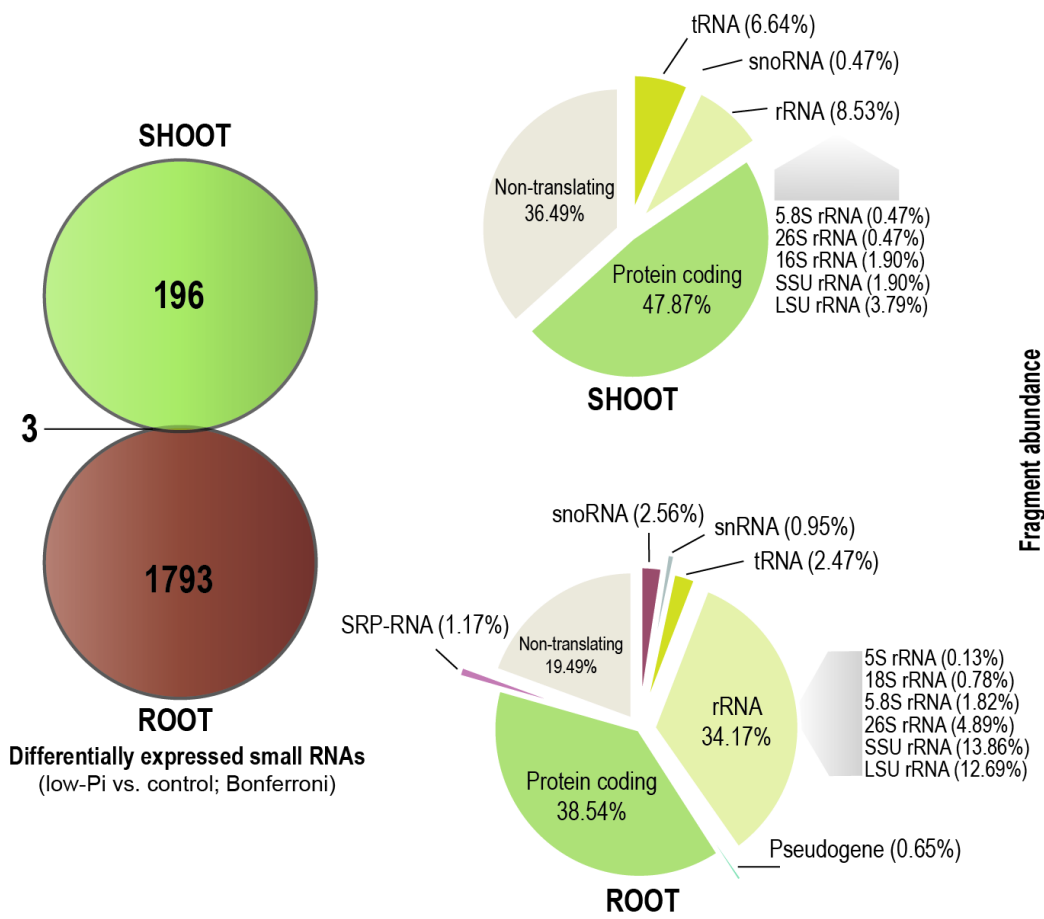

Shoot

sRNA (ID = 2117, $\left.\log _{2}(\mathrm{fc})=3.24\right)$ targets HORVU7Hr1G003920.11

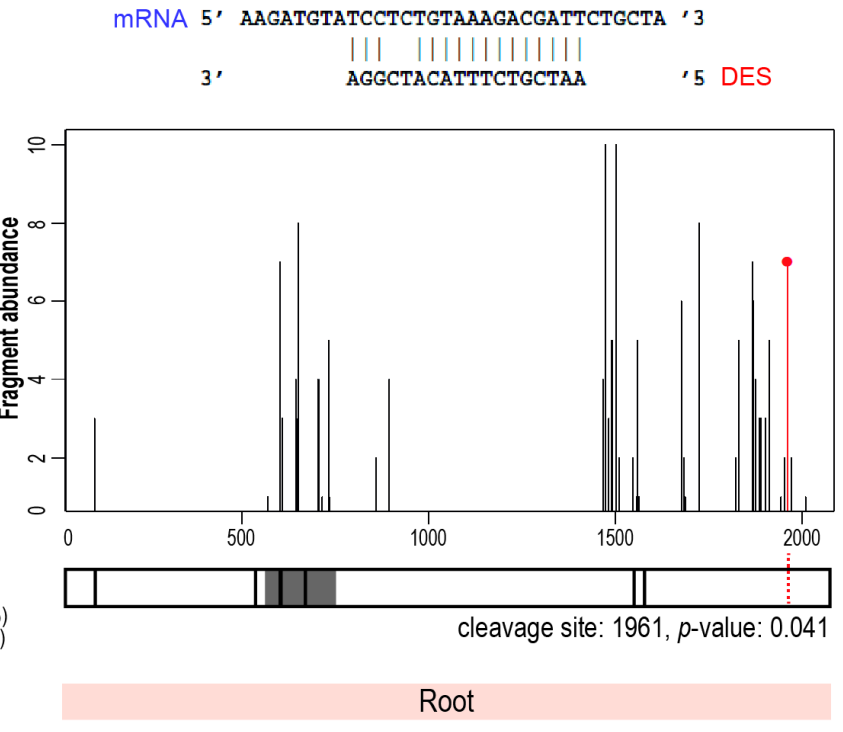

sRNA (ID $\left.=463, \log _{2}(\mathrm{fc})=-3.58\right)$ targets HORVU7Hr1G053570.3

MRNA 5' TTCGAGCCTCCACCAGAGTTTCCTCTGGCTTC ' 3

- |||||||||||||||||||| $\mid$

3' GCTCGGAGGTGGTCTCAAAGGAGA '5 DES

B
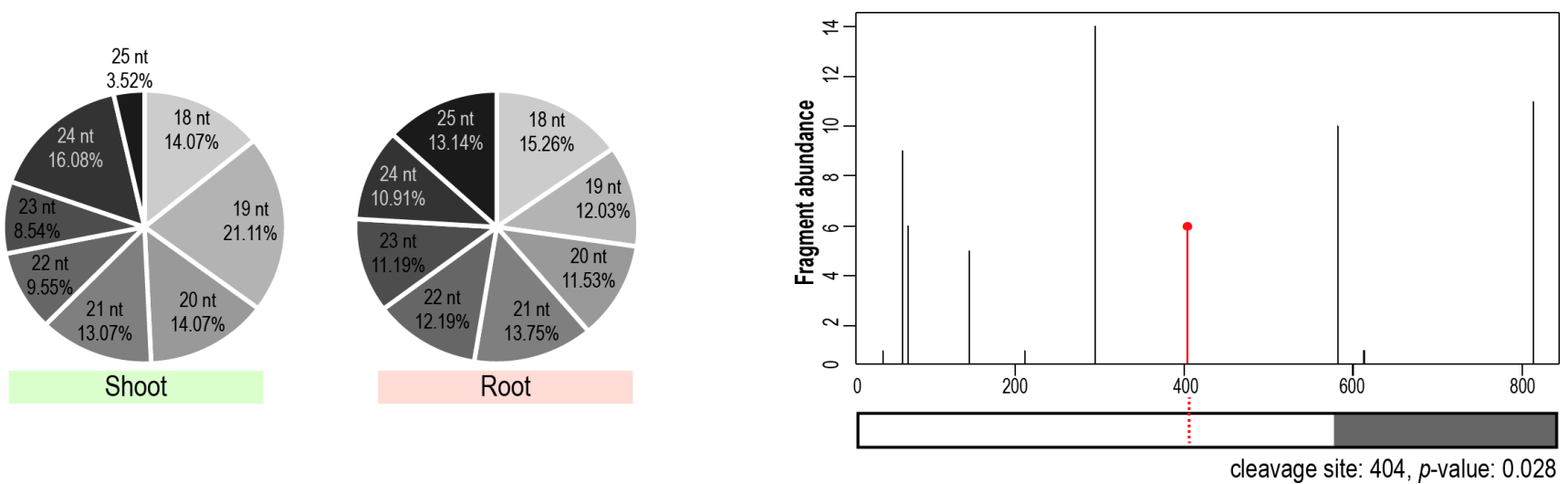

\section{Figure 4}

Differentially expressed other small RNAs (DESs) in barley plants under the low-Pi regime. (A) Venn's diagram illustrating the quantity of identified DESs with Bonferroni corrected p-value (left panel). The annotation distribution of DESs in barley shoots and roots based on the calculations present in Additional file 7 (right panel). (B) The length distribution of DESs in roots and shoots. (C) Degradome profiles identified two DESs that are significantly changed in shoot or root and exhibit high possibility to cleave mRNA targets. The HORVU7Hr1G053570 locus encodes an uncharacterized protein with unknown PTHR47188 domain; the HORVU7Hr1G003920 locus encodes glutaminyl-peptide cyclotransferase. The white boxes denote UTRs, the gray boxes denote coding sequence, and the red dotted line denote cleavage site. 
A
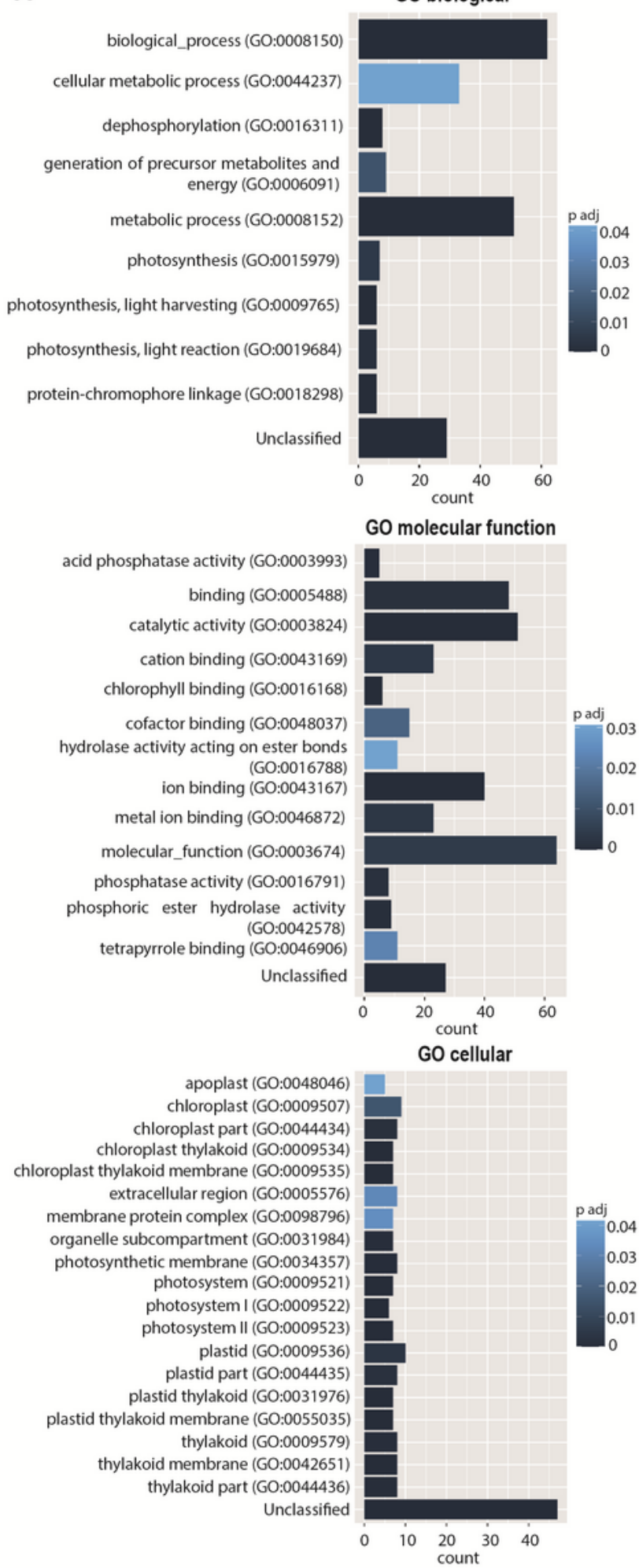

B

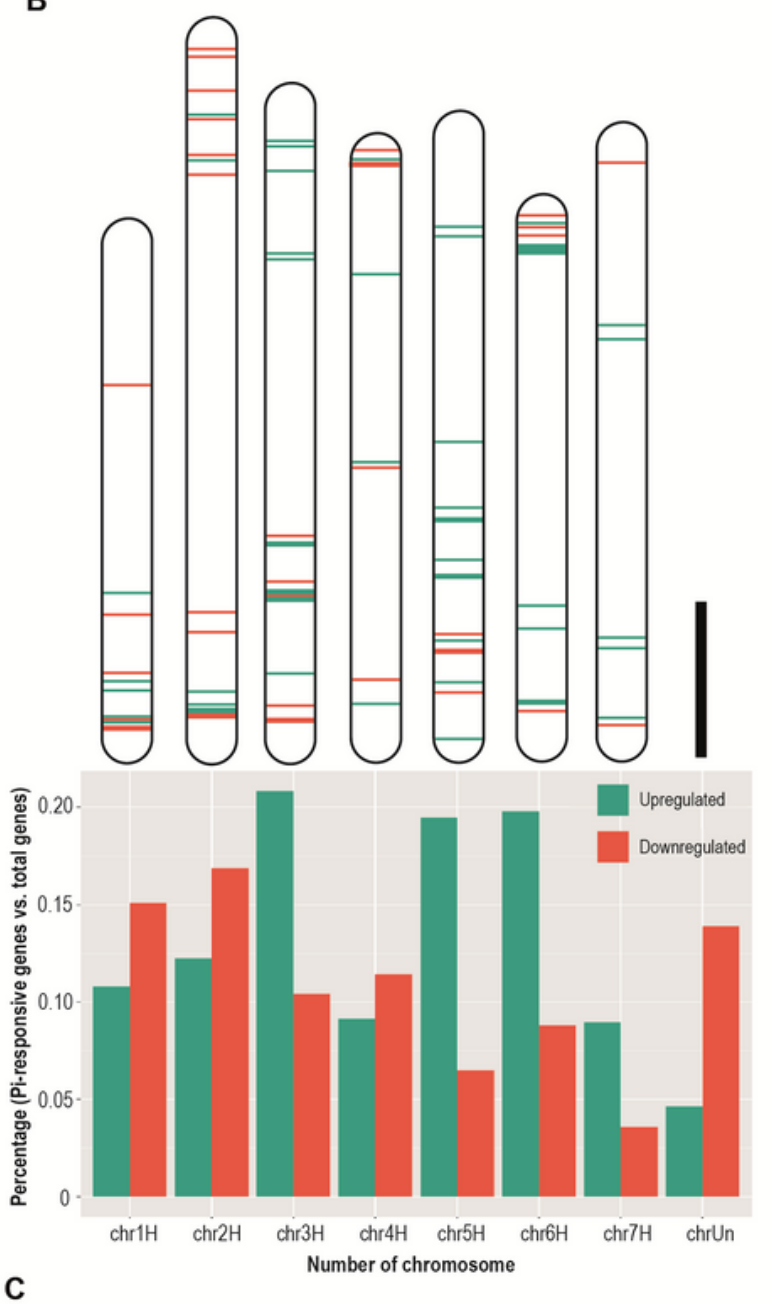

C

\begin{tabular}{|c|c|c|c|c|}
\hline 1 & chr4H & $\begin{array}{l}\log _{2} \mathrm{Fc} \\
14.20\end{array}$ & Encodes & $\begin{array}{l}\text { GO: biological process } \\
\text { Response to high light intensity }\end{array}$ \\
\hline 2 & chrUn & 13.35 & Mitochondrial-processing peptidase beta subunit & Targeting to mitochondrion \\
\hline 3 & chr1H & 8.90 & Chlorophyll a/b binding protein of LHCIl type & Photosynthesis \\
\hline 4 & chr6H & 7.30 & Alpha-amylase & Carbohydrate metabolism \\
\hline 5 & chr4 $4 \mathrm{H}$ & 5.89 & IPS1 & Pi signaling \\
\hline 6 & chr3H & 5.78 & GDPD1, chloroplasticlike & Lipid metabolism \\
\hline 7 & chr4 $4 \mathrm{H}$ & 5.27 & Probable monogalactosyldiacylglycerol synthase 2 & Glycolipid biosynthesis \\
\hline 8 & chr5H & 5.14 & Uncharacterized protein & N/A \\
\hline 9 & chr5H & 4.48 & O-fucosyltransferase 31-like isoform X2 & Fucose metabolism \\
\hline 10 & chr3H & 4.30 & Protein kinase domain-containing protein & Protein phosphorylation \\
\hline 89 & chr6H & -2.60 & NRT2.1 & Nitrate signaling \\
\hline 90 & chr1H & -2.61 & Pyridoxal phosphate-dependent transferase & Biosynthesis of amino acids \\
\hline 91 & chr4H & -2.62 & Oxalate oxidase & Defense response \\
\hline 92 & $\mathrm{chr} 2 \mathrm{H}$ & -2.62 & Cytochrome P450 & Oxidation-reduction process \\
\hline 93 & chr2H & -2.95 & Caleosin-like protein & Defense response \\
\hline 94 & chr1H & -3.05 & Chalcone synthase 2 & Stress response \\
\hline 95 & chrUn & -3.17 & Probable glutamate carboxypeptidase 2 & Stress response \\
\hline 96 & $\mathrm{chr} 2 \mathrm{H}$ & -3.41 & Beta-sesquiphellandrene synthase-like & Defense response \\
\hline 97 & chr4H & -4.41 & Oxalate oxidase 2 & Defense response \\
\hline 98 & $\mathrm{chr} 2 \mathrm{H}$ & -6.50 & Uncharacterized protein & N/A \\
\hline
\end{tabular}

Figure 5

Functional and genomic analysis of 98 differentially expressed genes in barley shoots under low-Pi. (A) The significantly enriched GO terms in the categories of biological process, molecular function, and cellular localization. The Bonferroni adjustment was applied to correct the p-values. (B) Chromosomal mapping of 98 DEGs identified in this study. Lower panel illustrates the percentage between quantitative distribution of either up-regulated or down-regulated genes under low-Pi conditions and total number of 
protein-coding genes in each barley chromosome. Scale bar for chromosomes $=160 \mathrm{Mbp}$. (C) Summarized list of ten most up-regulated and ten most down-regulated genes found in RNA-Seq analysis. Full list containing all 98 genes with their accession numbers is available in Additional file 8.

\section{A}

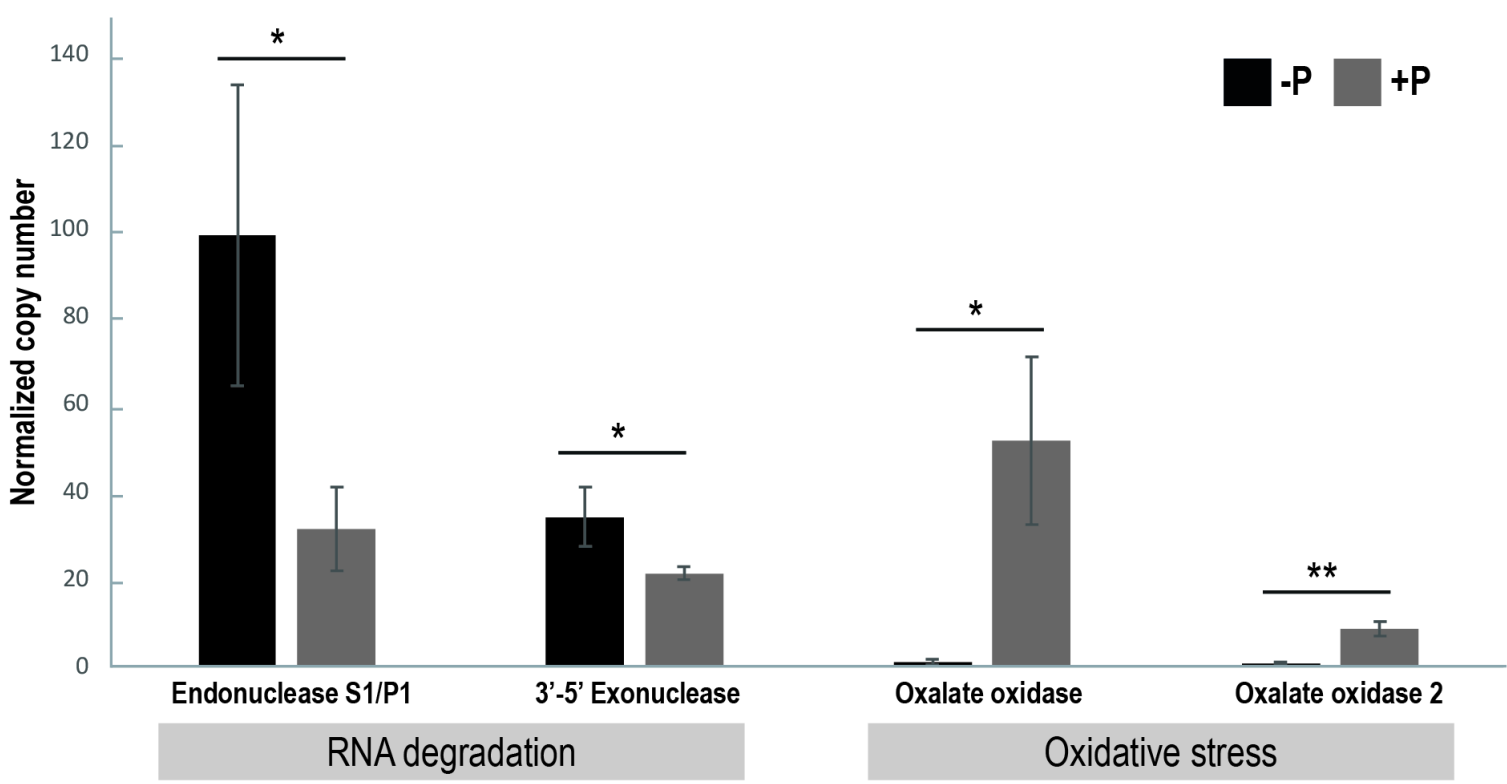

B

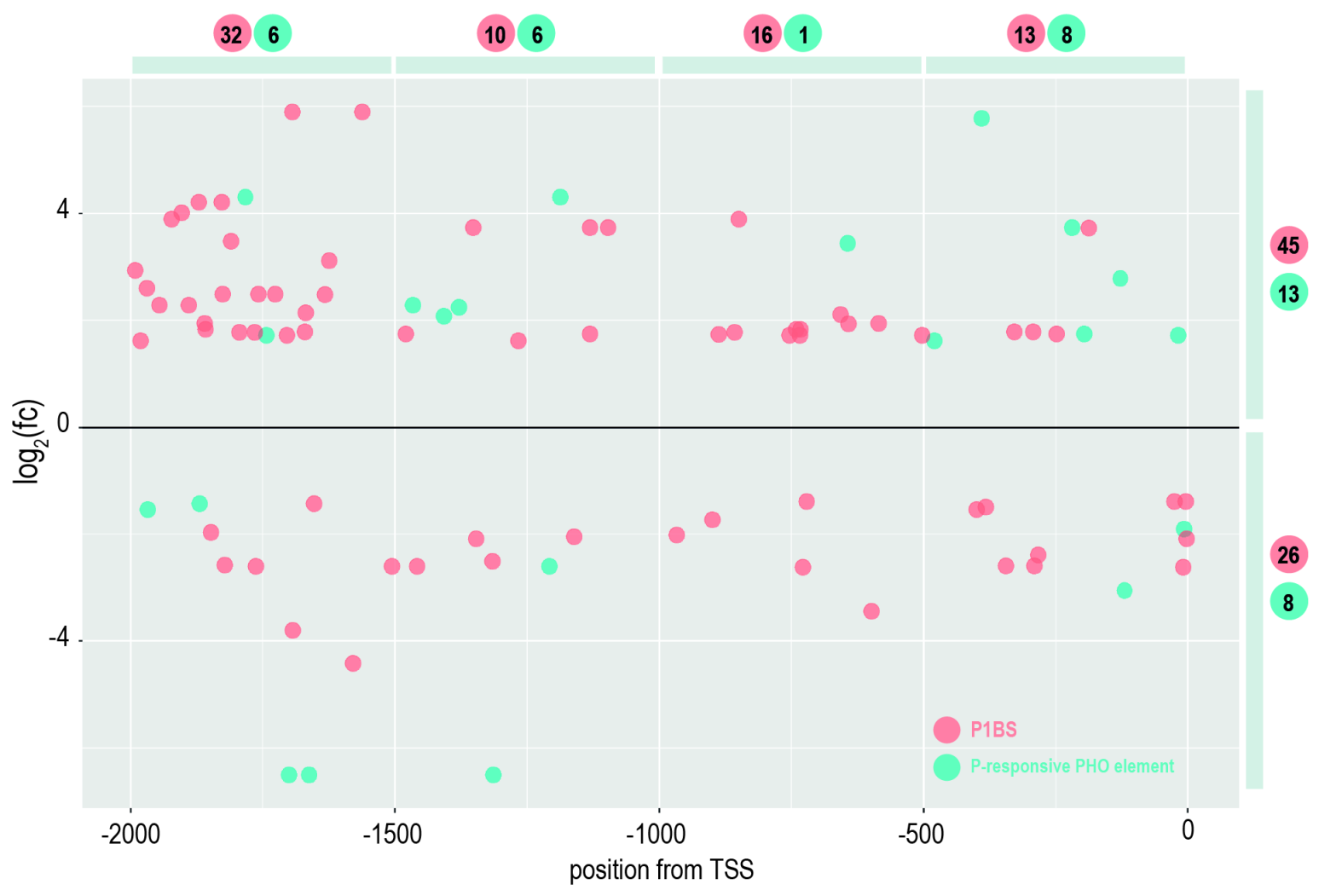

Figure 6

Molecular characterization of identified DEGs from barley shoot. (A) Quantification by ddPCR of the absolute expression levels of the DEGs belonging to two selected pathways in barley shoots. Two up- 
regulated DEGs, which encode endonuclease S1/P1 and 3'-5' exonuclease, are involved in nucleic acid metabolism and further RNA degradation. Two down-regulated DEGs, which encode two different oxalate oxidases, are involved in the reduction of oxidative stress. The bars represent copy numbers normalized to 1000 copies of the ARF1 reference gene; ${ }^{*} p$-value $<0.05$, ${ }^{*} p$-value $<0.001$, calculated using two-tailed Student's t-tests for three biological and two technical replicates. (B) Localization of all P1BS and Presponsive PHO cis-regulatory elements within the 2000 bp upstream from the DEG TSSs. On the graph, grouped motifs are specifically located in every $500 \mathrm{bp}$, induced $(\log 2(\mathrm{Fch})>0$ ), or repressed (log2(Fch) < 0 ). The motif quantity in each group is shown in either red (P1BS) or blue (PHO elements) dots.

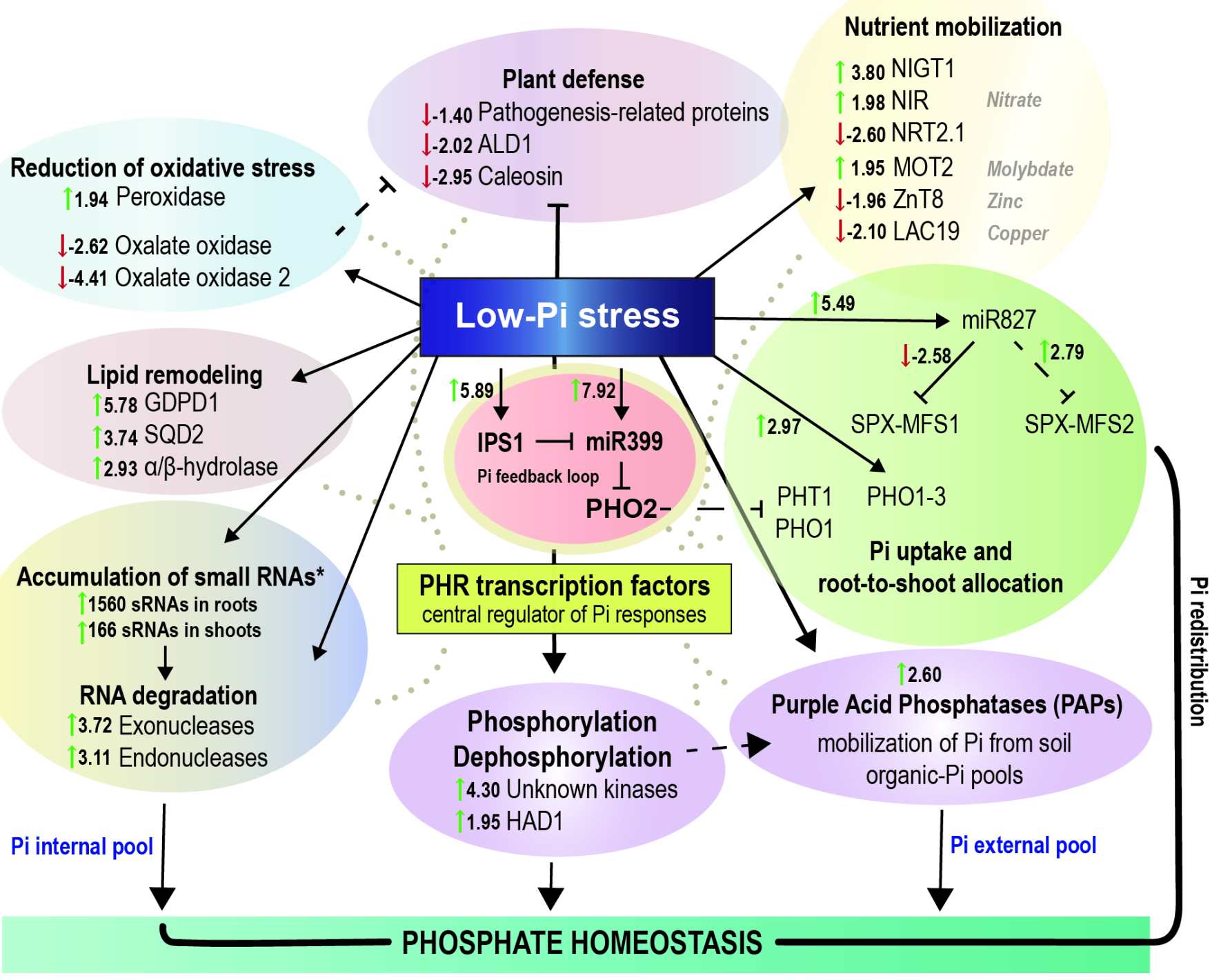

Figure 7

Barley pathways triggered by Pi-starvation to maintain plant homeostasis. Graphical overview illustrates primary strategies in Pi-starved barley plants based on our shoot transcriptomic analysis, small RNA-Seq, and degradome profiling. The low-Pi induced feedback loop is located in the middle part, which is involved in the positive regulation of phosphate transporters (i.e., PHT1, PH01) prompting Pi uptake. The MYB-coiled-coil (MYB-CC) protein family includes PHR transcription factors (yellow frame), which act as a major regulator to either induce or repress Pi-responsive genes in plants. The asterisk represents the 
data from shoot and root sRNA-Seq. Dotted lines display wide area of molecular networks, connecting most of the plant low-Pi stress responses. All components depicted on the graph are listed in Additional file 8. Values correspond to log2(fold change) with Bonferroni corrected p-values.

\section{Supplementary Files}

This is a list of supplementary files associated with this preprint. Click to download.

- Additionalfile1revised.pdf

- Additionalfile2.pdf

- Additionalfile3.xlsx

- Additionalfile4revised.xlsx

- Additionalfile5revised.xlsx

- Additionalfile6revised.pdf

- Additionalfile7revised.pdf

- Additionalfile8revised.pdf

- Additionalfile9revised.xlsx

- Additionalfile10revised.pdf

- Additionalfile11revised.pdf

- Additionalfile12revised.xlsx

- Additionalfile13revised.xlsx

- Additionalfile14revised.xlsx

- Additionalfile15revised.xlsx

- Additionalfile16revised.xlsx

- Additionalfile17revised.pdf

- Additionalfile18revised.xlsx

- Additionalfile19revised.pdf

- Additionalfile20revised.xlsx

- Additionalfile21 revised.pdf

- Additionalfile22revised.xIsx

- Additionalfile23revised.pdf

- Additionalfile24revised.pdf

- Additionalfile25.pdf

- Additionalfile26revised.jpg

- Additionalfile27revised.jpg

- MainTable1.pdf 
- MainTable2.pdf

- MainTable3.pdf

Page $42 / 42$ 